\title{
GREEN'S FUNCTIONS OF PANEITZ AND GJMS OPERATORS ON HYPERBOLIC SPACES AND SHARP HARDY-SOBOLEV-MAZ'YA INEQUALITIES ON HALF SPACES
}

\author{
GUOZHEN LU AND QIAOHUA YANG
}

\begin{abstract}
Using the Fourier analysis techniques on hyperbolic spaces and Green's function estimates, we confirm in this paper the conjecture given by the same authors in 43 . Namely, we prove that the sharp constant in the $\frac{n-1}{2}$-th order Hardy-Sobolev-Maz'ya inequality in the upper half space of dimension $n$ coincides with the best $\frac{n-1}{2}$-th order Sobolev constant when $n$ is odd and $n \geq 9$ (See Theorem [1.6). We will also establish a lower bound of the coefficient of the Hardy term for the $k$-th order Hardy-Sobolev-Maz'ya inequality in upper half space in the remaining cases of dimension $n$ and $k$-th order derivatives (see Theorem 1.7). Precise expressions and optimal bounds for Green's functions of the operator $-\Delta_{\mathbb{H}}-\frac{(n-1)^{2}}{4}$ on the hyperbolic space $\mathbb{B}^{n}$ and operators of the product form are given, where $\frac{(n-1)^{2}}{4}$ is the spectral gap for the Laplacian $-\Delta_{\mathbb{H}}$ on $\mathbb{B}^{n}$. Finally, we give the precise expression and optimal pointwise bound of Green's function of the Paneitz and GJMS operators on hyperbolic space, which are of their independent interest (see Theorem 1.10).
\end{abstract}

\section{INTRODUCTION}

The classical Sobolev inequalities and sharp constants play an essential role in analysis and geometry. If $k$ is a positive integer, then the $k$-th order Sobolev inequality states as follows (see [4, 50, 38, 14])

$$
\int_{\mathbb{R}^{n}}\left|(-\Delta)^{k / 2} u\right|^{2} d x \geq S_{n, k}\left(\int_{\mathbb{R}^{n}}|u|^{\frac{2 n}{n-2 k}} d x\right)^{\frac{n-2 k}{n}}, u \in C_{0}^{\infty}\left(\mathbb{R}^{n}\right), \quad 1 \leq k<\frac{n}{2},
$$

where

$$
S_{n, k}=\frac{\Gamma\left(\frac{n+2 k}{2}\right)}{\Gamma\left(\frac{n-2 k}{2}\right)} \omega_{n}^{2 k / n}
$$

is the best Sobolev constant and $\omega_{n}=\frac{2 \pi^{\frac{n+1}{2}}}{\Gamma\left(\frac{n+1}{2}\right)}$ is the surface measure of $\mathbb{S}^{n}=\left\{x \in \mathbb{R}^{n+1}:|x|=\right.$ 1\}. Maz'ya ([46], Section 2.1.6) gave a refinement of both the first order Sobolev and the

2000 Mathematics Subject Classification. Primary 35J20; 46 E35.

Key words and phrases. Green's function; Hyperbolic spaces; Hardy-Sobolev-Maz'ya inequalities, Paneitz and GJMS operators.

The first author was partly supported by a Simons collaboration grant from the Simons Foundation and the second author's research was partly supported by the National Natural Science Foundation of China (No.11201346). 
Hardy inequalities in half spaces which are known as the Hardy-Sobolev-Maz'ya inequality. It reads as follows

$$
\int_{\mathbb{R}_{+}^{n}}|\nabla u|^{2} d x-\frac{1}{4} \int_{\mathbb{R}_{+}^{n}} \frac{u^{2}}{x_{1}^{2}} d x \geq C_{n}\left(\int_{\mathbb{R}_{+}^{n}} x_{1}^{\gamma}|u|^{p} d x\right)^{\frac{2}{p}}, \quad u \in C_{0}^{\infty}\left(\mathbb{R}_{+}^{n}\right),
$$

where $2<p \leq \frac{2 n}{n-2}, \gamma=\frac{(n-2) p}{2}-n, \mathbb{R}_{+}^{n}=\left\{\left(x_{1}, x_{2}, \cdots, x_{n}\right) \in \mathbb{R}^{n}: x_{1}>0\right\}$ and $C_{n}$ is a positive constant which is independent of $u$. Such inequalities with a Hardy remaining term have been extensively studied by many authors (see e.g. [6, 8, 9, 10, 11, 12, 19, 20, 34, 44, 47, ,52] and the references therein).

It is known that the upper half space can be regarded as a hyperbolic space. Then the Hardy-Sobolev-Maz'ya inequality on the upper half space is equivalent to the corresponding inequality on the hyperbolic ball. To be more precise, we now recall the ball model as a hyperbolic space. The unit ball

$$
\mathbb{B}^{n}=\left\{x=\left(x_{1}, \cdots, x_{n}\right) \in \mathbb{R}^{n}|| x \mid<1\right\}
$$

equipped with the usual Poincaré metric

$$
d s^{2}=\frac{4\left(d x_{1}^{2}+\cdots+d x_{n}^{2}\right)}{\left(1-|x|^{2}\right)^{2}}
$$

is known as the hyperbolic space of ball model. The hyperbolic gradient is $\nabla_{\mathbb{H}}=\frac{1-|x|^{2}}{2} \nabla$ and the Laplace-Beltrami operator is given by

$$
\Delta_{\mathbb{H}}=\frac{1-|x|^{2}}{4}\left\{\left(1-|x|^{2}\right) \sum_{j=1}^{n} \frac{\partial^{2}}{\partial x_{j}^{2}}+2(n-2) \sum_{j=1}^{n} x_{j} \frac{\partial}{\partial x_{j}}\right\} .
$$

The hyperbolic volume is $d V=\left(\frac{2}{1-|x|^{2}}\right)^{n} d x$.

Then the GJMS operators on $\mathbb{B}^{n}$ is defined as follows (see [23], [21], [33])

$$
P_{k}=P_{1}\left(P_{1}+2\right) \cdots\left(P_{1}+k(k-1)\right), k \in \mathbb{N},
$$

where $P_{1}=-\Delta_{\mathbb{H}}-\frac{n(n-2)}{4}$ is the conformal Laplacian on $\mathbb{B}^{n}$ and

$$
P_{2}=\left(-\Delta_{\mathbb{H}}-\frac{n(n-2)}{4}\right)\left(-\Delta_{\mathbb{H}}-\frac{(n+2)(n-4)}{4}\right)
$$

is the well-known Paneitz operator (see [48]). The GJMS operator $P_{k}$ on $\mathbb{B}^{n}$ can also be written as

$$
P_{k}=\prod_{j=1}^{k}\left(-\Delta_{\mathbb{H}}-\frac{(n-1)^{2}}{4}+(j-1 / 2)^{2}\right) .
$$

Hebey proved in [25] (see also [26]) the following theorem: 
Theorem 1.1. Let $n \geq 4$. Suppose that there exists $\lambda \in \mathbb{R}$ such that for any $u \in C_{0}^{\infty}\left(\mathbb{B}^{n}\right)$,

$$
\int_{\mathbb{B}^{n}}\left(P_{1} u\right) u d V+\lambda \int_{\mathbb{B}^{n}} u^{2} d V \geq S_{n, 1}\left(\int_{\mathbb{B}^{n}}|u|^{\frac{2 n}{n-2}} d V\right)^{\frac{n-2}{n}},
$$

where $S_{n, 1}$ is the best first order Sobolev constant. Then $\lambda \geq 0$.

In case $n=3$, Benguria, Frank and Loss ([10]) proved the following

Theorem 1.2. There holds, for any $u \in C_{0}^{\infty}\left(\mathbb{B}^{n}\right)$,

$$
\int_{\mathbb{B}^{3}}\left(P_{1} u\right) u d V-\frac{1}{4} \int_{\mathbb{B}^{3}} u^{2} d V \geq S_{3,1}\left(\int_{\mathbb{B}^{n}}|u|^{6} d V\right)^{\frac{1}{3}}
$$

where $S_{3,1}$ is the best first order Sobolev constant.

From the above Theorem 1.2, we can see that the best constant $C_{n}$ in (1.3) is the same as the best sharp Sobolev constant for the first order Sobolev inequality when $n=3$. When $n \geq 4$, Theorem 1.1 shows that the sharp constant $C_{n}$ in (1.3) is strictly less than the Sobolev constant $S_{n, 1}$. On upper half space, The authors of [52] subsequently offered another proof of $C_{n}<S_{n, 1}$ when $n \geq 4$. Furthermore, they established the existence of an extremal function for the sharp constant $C_{n}$.

The sharp Sobolev inequalities on hyperbolic ball $\mathbb{B}^{n}$ read as follows (see Hebey [25] for $k=1$ and Liu [40] for $\left.2 \leq k<\frac{n}{2}\right)$ :

$$
\int_{\mathbb{B}^{n}}\left(P_{k} u\right) u d V \geq S_{n, k}\left(\int_{\mathbb{B}^{n}}|u|^{\frac{2 n}{n-2 k}} d V\right)^{\frac{n-2 k}{n}}, u \in C_{0}^{\infty}\left(\mathbb{B}^{n}\right), \quad 1 \leq k<\frac{n}{2},
$$

where $S_{n, k}$ is the best $k$-th order Sobolev constant. We remark that the above sharp Sobolev inequality on hyperbolic spaces (1.5) can also follow from the Sharp Sobolev inequality on spheres due to Beckner [5]. An alternative proof of (1.5) has been given in [43] (see Section 7 there).

The following higher order Hardy-Sobolev-Maz'ya inequalities have been recently established by the authors in [43] using Fourier analysis techniques on hyperbolic spaces.

Theorem 1.3. Let $2 \leq k<\frac{n}{2}$ and $2<p \leq \frac{2 n}{n-2 k}$. There exists a positive constant $C=$ $C(n, k, p)$ such that for each $u \in C_{0}^{\infty}\left(\mathbb{B}^{n}\right)$,

$$
\int_{\mathbb{B}^{n}}\left(P_{k} u\right) u d V-\prod_{i=1}^{k} \frac{(2 i-1)^{2}}{4} \int_{\mathbb{B}^{n}} u^{2} d V \geq C\left(\int_{\mathbb{B}^{n}}|u|^{p} d V\right)^{\frac{2}{p}} .
$$

This improves substantially the Poincaré-Sobolev inequalities (1.5) for higher order derivatives on the hyperbolic spaces $\mathbb{B}^{n}$ by subtracting a Hardy term on the left hand side. If $p=\frac{2 n}{n-2 k}$, then by (1.5), the sharp constant in (1.6) is less than or equal to the best $k$-th order Sobolev constant $S_{n, k}$.

As an application of Theorem 1.3, we have the following Hardy-Sobolev-Maz'ya inequalities for higher order derivatives (see [43]): 
Theorem 1.4. Let $2 \leq k<\frac{n}{2}$ and $2<p \leq \frac{2 n}{n-2 k}$. There exists a positive constant $C$ such that for each $u \in C_{0}^{\infty}\left(\mathbb{R}_{+}^{n}\right)$,

$$
\int_{\mathbb{R}_{+}^{n}}\left|\nabla^{k} u\right|^{2} d x-\prod_{i=1}^{k} \frac{(2 i-1)^{2}}{4} \int_{\mathbb{R}_{+}^{n}} \frac{u^{2}}{x_{1}^{2 k}} d x \geq C\left(\int_{\mathbb{R}_{+}^{n}} x_{1}^{\gamma}|u|^{p} d x\right)^{\frac{2}{p}},
$$

where $\gamma=\frac{(n-2 k) p}{2}-n$.

In terms of the Poincaré ball model $\mathbb{B}^{n}$, inequality (1.6) can be written as follows:

$$
\int_{\mathbb{B}^{n}}\left|\nabla^{k} u\right|^{2} d x-\prod_{i=1}^{k}(2 i-1)^{2} \int_{\mathbb{B}^{n}} \frac{u^{2}}{\left(1-|x|^{2}\right)^{2 k}} d x \geq C\left(\int_{\mathbb{B}^{n}}\left(1-|x|^{2}\right)^{\gamma}|u|^{p} d x\right)^{\frac{2}{p}} .
$$

Though it was not known in general yet whether the constant $C$ on the right hand sides of the inequalities (1.6), (1.7) and (1.8) in Theorems 1.3 and 1.4 respectively is the same as the best Sobolev constant $S_{n, k}$, we have established in [43] in the case of $n=5$ and $k=2$ that the best constant for the Hardy-Sobolev-Maz'ya inequality in (1.6), (1.7) and (1.8) coincides with the best Sobolev constant $S_{5,2}$. In fact, the following has been proved in [43].

Theorem 1.5. There holds, for each $u \in C_{0}^{\infty}\left(\mathbb{B}^{5}\right)$,

$$
\int_{\mathbb{B}^{5}}\left(P_{2} u\right) u d V-\frac{9}{16} \int_{\mathbb{B}^{5}} u^{2} d V \geq S_{5,2}\left(\int_{\mathbb{B}^{n}}|u|^{10} d V\right)^{\frac{1}{5}} .
$$

In terms of the Poincaré ball model $\mathbb{B}^{5}$ and the Poincaré half space model $\mathbb{H}^{5}$, respectively, inequality (1.9) is equivalent to the following:

$$
\begin{gathered}
\int_{\mathbb{B}^{5}}|\Delta f|^{2} d x-9 \int_{\mathbb{B}^{5}} \frac{f^{2}}{\left(1-|x|^{2}\right)^{4}} d x \geq S_{5,2}\left(\int_{\mathbb{B}^{5}}|f|^{10} d x\right)^{\frac{1}{5}}, f \in C_{0}^{\infty}\left(\mathbb{B}^{5}\right) ; \\
\int_{\mathbb{R}_{+}^{5}}|\Delta g|^{2} d x-\frac{9}{16} \int_{\mathbb{R}_{+}^{5}} \frac{g^{2}}{x_{1}^{4}} d x \geq S_{5,2}\left(\int_{\mathbb{R}_{+}^{5}}|g|^{10} d x\right)^{\frac{1}{5}}, g \in C_{0}^{\infty}\left(\mathbb{R}_{+}^{5}\right) .
\end{gathered}
$$

We also illustrated in [43] that the best constant for the Hardy-Sobolev-Maz'ya inequality does not coincide with the best Sobolev constant for $n=6$ and $k=2$ (see Remark 8.3 and Lemma 8.4 there). More recently, Hong has shown in [29] that the sharp constant of Poincaré-Sobolev inequality and the Hardy-Sobolev-Maz'ya inequality for $n=7$ and $k=3$ is also the same as the Sobolev constant $S_{7,3}$, by adapting the argument as done in [43] for the case $n=5$ and $k=2$.

We further conjectured in [43] that the sharp constant of Poincaré-Sobolev inequality and the Hardy-Sobolev-Maz'ya inequality for $n \geq 9$ and odd and $k=\frac{n-1}{2}$ also coincides with the Sobolev constant $S_{n, \frac{n-1}{2}}$.

One of the our main results in this paper is to confirm this conjecture and give a unified proof for $k=\frac{n-1}{2}$ and all $n \geq 5$ odd. 
Theorem 1.6. Let $n \geq 5$ be odd. There holds, for each $u \in C_{0}^{\infty}\left(\mathbb{B}^{n}\right)$,

$$
\int_{\mathbb{B}^{n}}\left(P_{(n-1) / 2} u\right) u d V-\prod_{j=1}^{(n-1) / 2} \frac{(2 j-1)^{2}}{4} \int_{\mathbb{B}^{n}} u^{2} d V \geq S_{n,(n-1) / 2}\left(\int_{\mathbb{B}^{n}}|u|^{2 n} d V\right)^{\frac{1}{n}} .
$$

In terms of the Poincaré ball model and the Poincaré half space model respectively, the inequality above is equivalent to the following:

$$
\begin{aligned}
& \int_{\mathbb{B}^{n}}\left|\nabla^{\frac{n-1}{2}} f\right|^{2} d x-\prod_{j=1}^{(n-1) / 2}(2 j-1)^{2} \int_{\mathbb{B}^{n}} \frac{f^{2}}{\left(1-|x|^{2}\right)^{n-1}} d x \geq S_{n,(n-1) / 2}\left(\int_{\mathbb{B}^{n}}|f|^{2 n} d x\right)^{\frac{1}{n}}, f \in C_{0}^{\infty}\left(\mathbb{B}^{n}\right) ; \\
& \int_{\mathbb{R}_{+}^{n}}\left|\nabla^{\frac{n-1}{2}} g\right|^{2} d x-\prod_{j=1}^{(n-1) / 2} \frac{(2 j-1)^{2}}{4} \int_{\mathbb{R}_{+}^{n}} \frac{g^{2}}{x_{1}^{n-1}} d x \geq S_{n,(n-1) / 2}\left(\int_{\mathbb{R}_{+}^{n}}|g|^{2 n} d x\right)^{\frac{1}{n}}, g \in C_{0}^{\infty}\left(\mathbb{R}_{+}^{n}\right) .
\end{aligned}
$$

Our second main result is:

Theorem 1.7. Let $2 \leq k<\frac{n}{2}$. Suppose that there exists $\lambda \in \mathbb{R}$ such that for any $u \in$ $C_{0}^{\infty}\left(\mathbb{B}^{n}\right)$,

$$
\int_{\mathbb{B}^{n}}\left(P_{k} u\right) u d V+\lambda \int_{\mathbb{B}^{n}} u^{2} d V \geq S_{n, k}\left(\int_{\mathbb{B}^{n}}|u|^{\frac{2 n}{n-2 k}} d V\right)^{\frac{n-2 k}{n}},
$$

where $S_{n, k}$ is the best $k$-th order Sobolev constant. Then we have the following:

(1). If $n \geq 4 k$, then $\lambda \geq 0$.

(2). If $2 k+2 \leq n<4 k$, then

$$
\lambda \geq-\frac{\Gamma(n / 2) \Gamma(k) \sum_{j=0}^{k-1} \frac{\Gamma\left(j+\frac{n-2 k}{2}\right)}{\Gamma(j+1) \Gamma\left(\frac{n-2 k}{2}\right)}}{2^{\frac{n+2 k}{2}} \Gamma\left(\frac{n-2 k}{2}\right) \int_{0}^{1}\left[r^{2 k-n}-\sum_{j=0}^{k-1} \frac{\Gamma\left(j+\frac{n-2 k}{2}\right)}{\Gamma(j+1) \Gamma\left(\frac{n-2 k}{2}\right)}\left(1-r^{2}\right)^{j}\right]^{2} \frac{r^{n-1} d r}{\left(1-r^{2}\right)^{2 k}}} .
$$

Remark 1.8. By Theorem 1.7, we have shown that if $n \geq 4 k$, then the sharp constant of $2 k$-th order Hardy-Sobolev-Maz'ya inequality is strictly less than $S_{n, k}$. In some cases of $2 k+2 \leq n<4 k$, we can also show that the sharp constant of $2 k$-th order Hardy-SobolevMaz'ya inequality is also strictly less than $S_{n, k}$. For instance, if $n=2 k+2$, we can compute the right hand of inequality $(1.10)$ is equal to $-\frac{(k-1)(k !)^{2}}{2^{2 k}}$, which is strictly greater than $-\prod_{j=1}^{k} \frac{(2 j-1)^{2}}{4}$. Similar results hold in the rest of the cases $n$, i. e. $2 k+2<n<4 k$. We leave it to the reader.

Remark 1.9. When $n=2 k+1$, i.e., $k=\frac{n-1}{2}$ and $n$ is odd, as shown in Theorem 1.6, the sharp constants for the Sobolev inequality and the Hardy-Sobolev-Maz'ya inequality are the same. When $n$ is even, and $k=\frac{n}{2}$, this is the borderline case, the Hardy-Adams inequalities hold as shown in [35], [36], [42] (see also [41], [53] for the Hardy-Trudinger-Moser inequality). 
Our next main results in this paper are the estimates of Green's functions of the Paneitz and GJMS operators on hyperbolic spaces which are of independent interests. It is well known that Green's function of the second order operator $P_{1}$

$$
P_{1}^{-1}=\frac{1}{\gamma_{n}(2)}\left[\left(\frac{1}{2 \sinh \frac{\rho}{2}}\right)^{n-2}-\left(\frac{1}{2 \cosh \frac{\rho}{2}}\right)^{n-2}\right]
$$

where $\gamma_{n}(\alpha)$ is defined by

$$
\gamma_{n}(\alpha)=\pi^{n / 2} 2^{\alpha} \frac{\Gamma(\alpha / 2)}{\Gamma\left(\frac{n-\alpha}{2}\right)}, \quad 0<\alpha<n
$$

and $\rho=\log \frac{1+|x|}{1-|x|}$ is the hyperbolic distance from $x \in \mathbb{B}^{n}$ to the origin.

We will establish the following precise expressions of the Green functions for the Paneitz operator $P_{2}$ and higher order GJMS operators $P_{k}$, which are of independent interest.

Theorem 1.10. Let $1<k<n / 2$. The Green's function of $P_{k}$ satisfies:

- if $n=2 m$, then

$$
P_{k}^{-1}(\rho)=\frac{4^{k-1}}{\gamma_{2 m}(2 k)(\sinh \rho)^{2 m-2}} \sum_{j=0}^{m-1-k} \frac{\Gamma(m)}{\Gamma(j+1) \Gamma(m-j)}\left(\sinh \frac{\rho}{2}\right)^{2 j+2 k-2} ;
$$

- if $n=2 m-1$, then

$$
P_{k}^{-1}(\rho)=\frac{4^{k-1} \sqrt{2}}{\gamma_{2 m}(2 k)} \sum_{j=0}^{m-1-k} \frac{\Gamma(m)}{\Gamma(j+1) \Gamma(m-j)} \int_{\rho}^{\infty}\left(\sinh \frac{r}{2}\right)^{2 j+2 k-2} \frac{(\sinh r)^{3-2 m}}{\sqrt{\cosh r-\cosh \rho}} d r .
$$

Furthermore, we have

$$
P_{k}^{-1}(\rho) \leq \frac{1}{\gamma_{n}(2 k)}\left[\left(\frac{1}{2 \sinh \frac{\rho}{2}}\right)^{n-2 k}-\left(\frac{1}{2 \cosh \frac{\rho}{2}}\right)^{n-2 k}\right], \quad \rho>0 .
$$

The organization of this paper is as follows. In Section 2, we will review some Fourier analysis on hyperbolic spaces that will be needed in the subsequent sections. Section 3 gives a precise expression of the Green function of the operator $\left(-\frac{(n-1)^{2}}{4}-\Delta_{\mathbb{H}}\right)^{-1}$ on hyperbolic spaces using the Legendre function of second type $Q_{\nu}^{\mu}(z)$. In Section 4 , when $n$ is odd and $n=2 m+1$ or when and $0 \leq k \leq m-1$, we give precise expressions for the Green functions of the operators $\left(k^{2}-(n-1)^{2} / 4-\Delta_{\mathbb{H}}\right)^{-1}$ and $\left[\prod_{j=0}^{l-1}\left((k+j)^{2}-(n-1)^{2} / 4-\Delta_{\mathbb{H}}\right)\right]^{-1}$ for $l \in\{1,2, \cdots, m\}$ and $k \in\{0,1, \cdots, m-l\}$. The proof of Theorem 1.6 is then given using these Green's functions and Fourier analysis on hyperbolic spaces. Section 5 gives the proof of Theorem 1.7. In Section 6, we provide the precise expressions of Green functions of the Paneitz and GJMS operators $P_{k}$ on hyperbolic spaces and offer an optimal pointwise bound of the Green functions. The proof of Theorem 1.10 is given in this section. 


\section{Notations AND PRELIMINARIES}

We begin by quoting some preliminary facts on Fourier analysis on hyperbolic spaces which will be needed in the sequel and refer to [1, 2, 3, 13, 15, 27, 28, 30, 31, 32, 39, 49, 51, 54] for more information about this subject.

2.1. The half space model $\mathbb{H}^{n}$. It is given by $\mathbb{R}_{+} \times \mathbb{R}^{n-1}=\left\{\left(x_{1}, \cdots, x_{n}\right): x_{1}>0\right\}$ equipped with the Riemannian metric $d s^{2}=\frac{d x_{1}^{2}+\cdots+d x_{n}^{2}}{x_{1}^{2}}$. The induced Riemannian measure can be written as $d V=\frac{d x}{x_{1}^{n}}$, where $d x$ is the Lebesgue measure on $\mathbb{R}^{n}$. The hyperbolic gradient is $\nabla_{\mathbb{H}}=x_{1} \nabla$ and the Laplace-Beltrami operator on $\mathbb{H}^{n}$ is given by

$$
\Delta_{\mathbb{H}}=x_{1}^{2} \Delta-(n-2) x_{1} \frac{\partial}{\partial x_{1}},
$$

where $\Delta=\sum_{i=1}^{n} \frac{\partial^{2}}{\partial x_{i}^{2}}$ is the Laplace operator on $\mathbb{R}^{n}$.

2.2. The ball model $\mathbb{B}^{n}$. Recall that it is given by the unit ball

$$
\mathbb{B}^{n}=\left\{x=\left(x_{1}, \cdots, x_{n}\right) \in \mathbb{R}^{n}|| x \mid<1\right\}
$$

equipped with the usual Poincaré metric

$$
d s^{2}=\frac{4\left(d x_{1}^{2}+\cdots+d x_{n}^{2}\right)}{\left(1-|x|^{2}\right)^{2}} .
$$

The hyperbolic gradient is $\nabla_{\mathbb{H}}=\frac{1-|x|^{2}}{2} \nabla$ and the Laplace-Beltrami operator is given by

$$
\Delta_{\mathbb{H}}=\frac{1-|x|^{2}}{4}\left\{\left(1-|x|^{2}\right) \sum_{i=1}^{n} \frac{\partial^{2}}{\partial x_{i}^{2}}+2(n-2) \sum_{i=1}^{n} x_{i} \frac{\partial}{\partial x_{i}}\right\} .
$$

Furthermore, the half space model $\mathbb{H}^{n}$ and the ball model $\mathbb{B}^{n}$ are equivalent.

2.3. Möbius transformations. For each $a \in \mathbb{B}^{n}$, we define the Möbius transformations $T_{a}$ by (see e.g. [1, 30])

$$
T_{a}(x)=\frac{|x-a|^{2} a-\left(1-|a|^{2}\right)(x-a)}{1-2 x \cdot a+|x|^{2}|a|^{2}},
$$

where $x \cdot a=x_{1} a_{1}+x_{2} a_{2}+\cdots+x_{n} a_{n}$ denotes the scalar product in $\mathbb{R}^{n}$. It is known that the measure on $\mathbb{B}^{n}$ is invariant with respect to the Möbius transformations.

Using the Möbius transformations, we can define the distance from $x$ to $y$ in $\mathbb{B}^{n}$ as follows

$$
\rho(x, y)=\rho\left(T_{x}(y)\right)=\rho\left(T_{y}(x)\right)=\log \frac{1+\left|T_{y}(x)\right|}{1-\left|T_{y}(x)\right|} .
$$

Also using the Möbius transformations, we can define the convolution of measurable functions $f$ and $g$ on $\mathbb{B}^{n}$ by

$$
(f * g)(x)=\int_{\mathbb{B}^{n}} f(y) g\left(T_{x}(y)\right) d V(y)
$$


provided this integral exists. It is easy to check that

$$
f * g=g * f \text {. }
$$

Furthermore, if $g$ is radial, i.e. $g=g(\rho)$, then

$$
(f * g) * h=f *(g * h)
$$

provided $f, g, h \in L^{1}\left(\mathbb{B}^{n}\right)$

2.4. The Fourier transform on hyperbolic spaces. In term of the ball model, we define the Fourier transform on hyperbolic spaces as follows. Set

$$
e_{\lambda, \zeta}(x)=\left(\frac{\sqrt{1-|x|^{2}}}{|x-\zeta|}\right)^{n-1+i \lambda}, x \in \mathbb{B}^{n}, \quad \lambda \in \mathbb{R}, \quad \zeta \in \mathbb{S}^{n-1} .
$$

The Fourier transform of a function $f$ on $\mathbb{B}^{n}$ can be defined as

$$
\widehat{f}(\lambda, \zeta)=\int_{\mathbb{B}^{n}} f(x) e_{-\lambda, \zeta}(x) d V
$$

provided this integral exists. If $g \in C_{0}^{\infty}\left(\mathbb{B}^{n}\right)$ is radial, then

$$
\widehat{(f * g)}=\widehat{f} \cdot \widehat{g} \text {. }
$$

Moreover, the following inversion formula holds for $f \in C_{0}^{\infty}\left(\mathbb{B}^{n}\right)$ :

$$
f(x)=D_{n} \int_{-\infty}^{+\infty} \int_{\mathbb{S}^{n-1}} \widehat{f}(\lambda, \zeta) e_{\lambda, \zeta}(x)|\mathfrak{c}(\lambda)|^{-2} d \lambda d \sigma(\varsigma),
$$

where $D_{n}=\frac{1}{2^{3-n} \pi\left|\mathbb{S}^{n-1}\right|}$ and $\mathfrak{c}(\lambda)$ is the Harish-Chandra $\mathfrak{c}$-function given by

$$
\mathfrak{c}(\lambda)=\frac{2^{n-1-i \lambda} \Gamma(n / 2) \Gamma(i \lambda)}{\Gamma\left(\frac{n-1+i \lambda}{2}\right) \Gamma\left(\frac{1+i \lambda}{2}\right)} .
$$

Similarly, there holds the Plancherel formula:

$$
\int_{\mathbb{B}^{n}}|f(x)|^{2} d V=D_{n} \int_{-\infty}^{+\infty} \int_{\mathbb{S}^{n-1}}|\widehat{f}(\lambda, \zeta)|^{2}|\mathfrak{c}(\lambda)|^{-2} d \lambda d \sigma(\varsigma) .
$$

Since $e_{\lambda, \zeta}(x)$ is an eigenfunction of $\Delta_{\mathbb{H}}$ with eigenvalue $-\frac{(n-1)^{2}+\lambda^{2}}{4}$, it is easy to check that, for $f \in C_{0}^{\infty}\left(\mathbb{B}^{n}\right)$,

$$
\widehat{\Delta_{\mathbb{H}} f}(\lambda, \zeta)=-\frac{(n-1)^{2}+\lambda^{2}}{4} \widehat{f}(\lambda, \zeta) .
$$

Therefore, in analogy with the Euclidean setting, we define the fractional Laplacian on hyperbolic space as follows:

$$
\left(\widehat{\left.-\Delta_{\mathbb{H}}\right)^{\gamma}} f(\lambda, \zeta)=\left(\frac{(n-1)^{2}+\lambda^{2}}{4}\right)^{\gamma} \widehat{f}(\lambda, \zeta), \quad \gamma \in \mathbb{R} .\right.
$$




\section{Precise expression And estimate for Green's function of $\left(-\frac{(n-1)^{2}}{4}-\Delta_{\mathbb{H}}\right)^{-1}$}

In this section, we give a precise expression and estimate of the kernel of Green's function of the operator $\left(-(n-1)^{2} / 4-\Delta_{\mathbb{H}}\right)^{-1}$, where $(n-1)^{2} / 4$ is the spectral gap of the Laplacian $-\Delta_{\mathbb{H}}$ on the hyperbolic ball $\mathbb{B}^{n}$. We will need some facts about the Legendre function of second type $Q_{\nu}^{\mu}(z)$, which is defined by (see [18])

$$
\begin{gathered}
Q_{\nu}^{\mu}(z)=e^{i(\pi \mu)} 2^{-\nu-1} \frac{\Gamma(\nu+\mu+1)}{\Gamma(\nu+1)}\left(z^{2}-1\right)^{-\mu / 2} \int_{0}^{\pi}(z+\cos t)^{\mu-\nu-1}(\sin t)^{2 \nu+1} d t \\
\operatorname{Re\nu }>-1, \quad \operatorname{Re}(\nu+\mu+1)>0 .
\end{gathered}
$$

It is known that (see [18])

$$
\begin{gathered}
Q_{\nu}^{\mu}(\cosh \rho)=e^{i(\pi \mu)} \frac{\sqrt{\pi}}{\sqrt{2}} \frac{\sinh ^{\mu} \rho}{\Gamma(1 / 2-\mu)} \int_{\rho}^{\infty} e^{-\left(\nu+\frac{1}{2}\right) r}(\cosh r-\cosh \rho)^{-\mu-1 / 2} d r, \\
\rho>0, \quad \operatorname{Re}(\nu+\mu+1)>0, \quad \operatorname{Re} \mu<1 / 2 .
\end{gathered}
$$

and (see [22])

$$
\begin{aligned}
\int_{u}^{\infty}\left(x^{2}-1\right)^{\frac{1}{2} \lambda}(x-u)^{\mu-1} Q_{\nu}^{-\lambda}(x) d x= & \Gamma(\mu) e^{\mu \pi i}\left(u^{2}-1\right)^{\frac{1}{2} \lambda+\frac{1}{2} \mu} Q_{\nu}^{-\lambda-\mu}(u), \\
& |\arg (u-1)|<\pi, \quad 0<\operatorname{Re} \mu<1+\operatorname{Re}(\nu-\lambda) .
\end{aligned}
$$

Setting $z=\cosh \rho$ and using (3.1), we have

$$
Q_{\nu}^{\mu}(\cosh \rho)=e^{i(\pi \mu)} 2^{-\nu-1} \frac{\Gamma(\nu+\mu+1)}{\Gamma(\nu+1)} \sinh ^{-\mu} \rho \int_{0}^{\pi}(\cosh \rho+\cos t)^{\mu-\nu-1}(\sin t)^{2 \nu+1} d t
$$

Setting $u=\cosh \rho$ and using (3.3), we have

$$
\int_{\rho}^{\infty} \frac{(\sinh r)^{\lambda+1}}{(\cosh r-\cosh \rho)^{1-\mu}} Q_{\nu}^{-\lambda}(\cosh r) d r=\Gamma(\mu) e^{\mu \pi i}(\sinh \rho)^{\lambda+\mu} Q_{\nu}^{-\lambda-\mu}(\cosh \rho) .
$$

Let $n \geq 2$. From now on, we will denote by $e^{t \Delta_{\mathbb{H}}}$ the heat kernel on $\mathbb{B}^{n}$. It is well known that $e^{t \Delta_{\mathbb{H}}}$ depends only on $t$ and $\rho(x, y)$. In fact, $e^{t \Delta_{\mathbb{H}}}$ is given explicitly by the following formulas (see e.g. [16, 24]):

- If $n=2 m$, then

$$
\begin{aligned}
e^{t \Delta_{\mathbb{H}}} & =(2 \pi)^{-\frac{n+1}{2}} t^{-\frac{1}{2}} e^{-\frac{(n-1)^{2}}{4} t} \int_{\rho}^{+\infty} \frac{\sinh r}{\sqrt{\cosh r-\cosh \rho}}\left(-\frac{1}{\sinh r} \frac{\partial}{\partial r}\right)^{m} e^{-\frac{r^{2}}{4 t}} d r \\
& =\frac{1}{2(2 \pi)^{\frac{n+1}{2}}} t^{-\frac{3}{2}} \int_{\rho}^{+\infty} \frac{\sinh r}{\sqrt{\cosh r-\cosh \rho}}\left(-\frac{1}{\sinh r} \frac{\partial}{\partial r}\right)^{m-1}\left(\frac{r}{\sinh r} e^{-\frac{r^{2}}{4 t}}\right) d r
\end{aligned}
$$


- If $n=2 m+1$, then

$$
\begin{aligned}
e^{t \Delta_{\mathbb{H}}} & =2^{-m-1} \pi^{-m-1 / 2} t^{-\frac{1}{2}} e^{-\frac{(n-1)^{2}}{4} t}\left(-\frac{1}{\sinh \rho} \frac{\partial}{\partial \rho}\right)^{m} e^{-\frac{\rho^{2}}{4 t}} \\
& =2^{-m-2} \pi^{-m-1 / 2} t^{-\frac{3}{2}} e^{-\frac{(n-1)^{2}}{4} t}\left(-\frac{1}{\sinh \rho} \frac{\partial}{\partial \rho}\right)^{m-1}\left(\frac{\rho}{\sinh \rho} e^{-\frac{\rho^{2}}{4 t}}\right) .
\end{aligned}
$$

An explicit expression of Green's function $\left(\lambda-\Delta_{\mathbb{H}}\right)^{-1}$ with $\lambda>-\frac{(n-1)^{2}}{4}$ is given by (see [45] for $\lambda \geq 0$ and [37] for $\left.\lambda>-\frac{(n-1)^{2}}{4}\right)$

$$
\left(\lambda-\Delta_{\mathbb{H}}\right)^{-1}=(2 \pi)^{-\frac{n}{2}}(\sinh \rho)^{-\frac{n-2}{2}} e^{-\frac{(n-2) \pi}{2} i} Q_{\theta_{n}(\lambda)}^{\frac{n-2}{2}}(\cosh \rho), \quad n \geq 3 .
$$

where

$$
\theta_{n}(\lambda)=\sqrt{\lambda+\frac{(n-1)^{2}}{4}}-\frac{1}{2}
$$

We shall show that (3.8) is also valid for $\lambda=-\frac{(n-1)^{2}}{4}$. Firstly, we prove the following Lemma:

Lemma 3.1. Let $m$ be a positive integer. Then we have

$$
\left(-\frac{1}{\sinh \rho} \frac{\partial}{\partial \rho}\right)^{m-1} \frac{1}{\sinh \rho}=\frac{\Gamma(m)}{\pi} \cdot \frac{1}{(\sinh \rho)^{2 m-1}} \int_{0}^{\pi}(\cosh \rho+\cos t)^{m-1} d t, \quad \rho>0 .
$$

Proof. We shall prove it by induction. It is easy to check (3.10) is valid for $m=1$ and $m=2$. Assume (3.10) is valid for $m=k \geq 2$. Then for $m=k+1$, we have

$$
\begin{aligned}
\left(-\frac{1}{\sinh \rho} \frac{\partial}{\partial \rho}\right)^{k} \frac{1}{\sinh \rho}= & \frac{\Gamma(k)}{\pi}\left(-\frac{1}{\sinh \rho} \frac{\partial}{\partial \rho}\right) \frac{1}{(\sinh \rho)^{2 k-1}} \int_{0}^{\pi}(\cosh \rho+\cos t)^{k-1} d t \\
= & \frac{\Gamma(k)}{\pi} \frac{1}{(\sinh \rho)^{2 k+1}}\left[(2 k-1) \cosh \rho \int_{0}^{\pi}(\cosh \rho+\cos t)^{k-1} d t-\right. \\
& \left.(k-1) \sinh ^{2} \rho \int_{0}^{\pi}(\cosh \rho+\cos t)^{k-2} d t\right] .
\end{aligned}
$$

To finish the proof, it is enough to show

$$
\begin{aligned}
& (2 k-1) \cosh \rho \int_{0}^{\pi}(\cosh \rho+\cos t)^{k-1} d t-(k-1) \sinh ^{2} \rho \int_{0}^{\pi}(\cosh \rho+\cos t)^{k-2} d t \\
= & k \int_{0}^{\pi}(\cosh \rho+\cos t)^{k} d t .
\end{aligned}
$$


In fact,

$$
\begin{aligned}
& (2 k-1) \cosh \rho \int_{0}^{\pi}(\cosh \rho+\cos t)^{k-1} d t-(k-1) \sinh ^{2} \rho \int_{0}^{\pi}(\cosh \rho+\cos t)^{k-2} d t \\
& -k \int_{0}^{\pi}(\cosh \rho+\cos t)^{k} d t \\
= & \int_{0}^{\pi}(\cosh \rho+\cos t)^{k-2}\left[(2 k-1) \cosh \rho(\cosh \rho+\cos t)-(k-1) \sinh ^{2} \rho-k(\cosh \rho+\cos t)^{2}\right] d t \\
= & \int_{0}^{\pi}(\cosh \rho+\cos t)^{k-2}\left[(k-1) \sin ^{2} t-\cos t(\cosh \rho+\cos t)\right] d t \\
= & (k-1) \int_{0}^{\pi}(\cosh \rho+\cos t)^{k-2} \sin ^{2} t d t-\int_{0}^{\pi}(\cosh \rho+\cos t)^{k-1} \cos t d t d t=0 .
\end{aligned}
$$

This completes the proof of Lemma 3.1 .

Now we show that (3.8) is valid for $\lambda=-\frac{(n-1)^{2}}{4}$.

Lemma 3.2. There holds, for $n \geq 3$ and $\rho>0$,

$$
\left(-(n-1)^{2} / 4-\Delta_{\mathbb{H}}\right)^{-1}=(2 \pi)^{-\frac{n}{2}}(\sinh \rho)^{-\frac{n-2}{2}} e^{-\frac{(n-2) \pi}{2} i} Q_{-1 / 2}^{\frac{n-2}{2}}(\cosh \rho) .
$$

Proof. If $n=2 m+1$, then by (3.7), (3.10) and (3.4), we have

$$
\begin{aligned}
\left(-(n-1)^{2} / 4-\Delta_{\mathbb{H}}\right)^{-1} & =\int_{0}^{\infty} e^{t\left(\Delta_{\mathbb{H}}+\frac{(n-1)^{2}}{4}\right)} d t \\
& =2^{-m-2} \pi^{-m-1 / 2}\left(-\frac{1}{\sinh \rho} \frac{\partial}{\partial \rho}\right)^{m-1}\left(\frac{\rho}{\sinh \rho} \int_{0}^{\infty} t^{-\frac{3}{2}} e^{-\frac{\rho^{2}}{4 t}} d t\right) \\
& =2^{-m-1} \pi^{-m}\left(-\frac{1}{\sinh \rho} \frac{\partial}{\partial \rho}\right)^{m-1} \frac{1}{\sinh \rho} \\
& =\frac{\Gamma(m)}{(2 \pi)^{m+1}} \cdot \frac{1}{(\sinh \rho)^{2 m-1}} \int_{0}^{\pi}(\cosh \rho+\cos t)^{m-1} d t \\
& =\frac{\Gamma\left(\frac{n-1}{2}\right)}{(2 \pi)^{\frac{n+1}{2}}} \cdot \frac{1}{(\sinh \rho)^{n-2}} \int_{0}^{\pi}(\cosh \rho+\cos t)^{\frac{n-3}{2}} d t \\
& =(2 \pi)^{-\frac{n}{2}}(\sinh \rho)^{-\frac{n-2}{2}} e^{-\frac{(n-2) \pi}{2} i} Q_{-1 / 2}^{(n-2) / 2}(\cosh \rho) .
\end{aligned}
$$


If $n=2 m$, then by (3.6), (3.10), (3.4) and (3.5), we have

$$
\begin{aligned}
& \left(-\Delta_{\mathbb{H}}-(n-1)^{2} / 4\right)^{-1}=\int_{0}^{\infty} e^{t\left(\Delta_{\mathbb{H}}+\frac{(n-1)^{2}}{4}\right)} d t \\
= & \frac{1}{2(2 \pi)^{\frac{n+1}{2}}} \int_{\rho}^{+\infty} \frac{\sinh r}{\sqrt{\cosh r-\cosh \rho}}\left(-\frac{1}{\sinh r} \frac{\partial}{\partial r}\right)^{m-1}\left(\frac{r}{\sinh r} \int_{0}^{\infty} t^{-\frac{3}{2}} e^{-\frac{r^{2}}{4 t}} d t\right) d r \\
= & \frac{\sqrt{\pi}}{(2 \pi)^{\frac{n+1}{2}}} \int_{\rho}^{+\infty} \frac{\sinh r}{\sqrt{\cosh r-\cosh \rho}}\left(-\frac{1}{\sinh r} \frac{\partial}{\partial r}\right)^{m-1} \frac{1}{\sinh r} d r \\
= & \frac{\sqrt{\pi}}{(2 \pi)^{\frac{n+1}{2}}} \int_{\rho}^{+\infty} \frac{\sinh r}{\sqrt{\cosh r-\cosh \rho}}\left[\frac{\Gamma(m)}{\pi} \cdot \frac{1}{(\sinh r)^{2 m-1}} \int_{0}^{\pi}(\cosh r+\cos t)^{m-1} d t\right] d r \\
= & \frac{\sqrt{\pi}}{(2 \pi)^{\frac{n+1}{2}}} \int_{\rho}^{+\infty} \frac{(\sinh r)^{-\frac{2 m-3}{2}}}{\sqrt{\cosh r-\cosh \rho}} \sqrt{\frac{2}{\pi}} e^{-\frac{(2 m-1) \pi}{2} i} Q_{-1 / 2}^{(2 m-1) / 2}(\cosh r) d r \\
= & \frac{\sqrt{2}}{(2 \pi)^{\frac{n+1}{2}}} e^{-\frac{(2 m-1) \pi}{2} i} \int_{\rho}^{+\infty} \frac{(\sinh r)^{-\frac{2 m-3}{2}}}{\sqrt{\cosh r-\cosh \rho}} Q_{-1 / 2}^{(2 m-1) / 2}(\cosh r) d r \\
= & \frac{\sqrt{2}}{(2 \pi)^{\frac{n+1}{2}}} e^{-\frac{(2 m-1) \pi}{2} i} \cdot \Gamma(1 / 2) e^{\frac{\pi i}{2}}(\sinh \rho)^{1-m} Q_{-1 / 2}^{m-1}(\cosh \rho) \\
= & (2 \pi)^{-\frac{n}{2}}(\sinh \rho)^{-\frac{n-2}{2}} e^{-\frac{(n-2) \pi}{2} i} Q_{-1 / 2}^{(n-2) / 2}(\cosh \rho) .
\end{aligned}
$$

The proof of Lemma 3.2 is thereby completed.

Therefore, for $n \geq 3$ and $\lambda=\nu^{2}-(n-1)^{2} / 4$ with $\nu \geq 0$, we have, by Lemma 3.2 and (3.4),

$$
\begin{aligned}
& \left(\nu^{2}-(n-1)^{2} / 4-\Delta_{\mathbb{H}}\right)^{-1}=(2 \pi)^{-\frac{n}{2}}(\sinh \rho)^{-\frac{n-2}{2}} e^{-\frac{(n-2) \pi}{2} i} Q_{\nu-\frac{1}{2}}^{\frac{n-2}{2}}(\cosh \rho) \\
= & \frac{(2 \pi)^{-\frac{n}{2}} \Gamma\left(\frac{n-1}{2}+\nu\right)}{2^{\nu+\frac{1}{2}} \Gamma\left(\nu+\frac{1}{2}\right)}(\sinh \rho)^{2-n} \int_{0}^{\pi}(\cosh \rho+\cos t)^{\frac{n-3}{2}-\nu}(\sin t)^{2 \nu} d t .
\end{aligned}
$$

\section{More Green's function estimates and Proof of Theorem 1.6}

In this section, when $n=2 m+1$ and $0 \leq k \leq m-1$, we will give precise expressions for the Green functions of the operators $\left(k^{2}-(n-1)^{2} / 4-\Delta_{\mathbb{H}}\right)^{-1}$ and $\left[\prod_{j=0}^{l-1}\left((k+j)^{2}-(n-1)^{2} / 4-\Delta_{\mathbb{H}}\right)\right]^{-1}$ for $l \in\{1,2, \cdots, m\}$ and $k \in\{0,1, \cdots, m-l\}$. These estimates play an important role in our proof of Theorem 1.6.

Lemma 4.1. If $n=2 m+1$ and $0 \leq k \leq m-1$, then

$$
\begin{aligned}
& \left(k^{2}-(n-1)^{2} / 4-\Delta_{\mathbb{H}}\right)^{-1} \\
= & \frac{1}{4 \pi^{m+\frac{1}{2}}(\sinh \rho)^{2 m-1}} \sum_{j=0}^{m-k-1} \frac{\Gamma(m+k) \Gamma(m-k) \Gamma\left(m-j-\frac{1}{2}\right)}{\Gamma(j+1) \Gamma(m-k-j) \Gamma(m+k-j)}\left(\sinh \frac{\rho}{2}\right)^{2 j} .
\end{aligned}
$$


Proof. If $n=2 m+1$ and $0 \leq k \leq m-1$, then by (3.12) and binomial theorem, we have

$$
\begin{aligned}
& \left(k^{2}-(n-1)^{2} / 4-\Delta_{\mathbb{H}}\right)^{-1} \\
= & \frac{(2 \pi)^{-\frac{n}{2}} \Gamma(m+k)}{2^{k+\frac{1}{2}} \Gamma\left(k+\frac{1}{2}\right)}(\sinh \rho)^{1-2 m} \int_{0}^{\pi}(\cosh \rho+\cos t)^{m-1-k}(\sin t)^{2 k} d t \\
= & \frac{(2 \pi)^{-\frac{n}{2}} \Gamma(m+k)}{2^{k+\frac{1}{2}} \Gamma\left(k+\frac{1}{2}\right)}(\sinh \rho)^{1-2 m} \int_{0}^{\pi}\left(2 \sinh ^{2} \frac{\rho}{2}+1+\cos t\right)^{m-1-k}(\sin t)^{2 k} d t \\
= & \frac{(2 \pi)^{-\frac{n}{2}} \Gamma(m+k)}{2^{k+\frac{1}{2}} \Gamma\left(k+\frac{1}{2}\right)}(\sinh \rho)^{1-2 m} \sum_{j=0}^{m-1-k} C_{m-1-k}^{j}\left(2 \sinh ^{2} \frac{\rho}{2}\right)^{j} \int_{0}^{\pi}(1+\cos t)^{m-1-k-j}(\sin t)^{2 k} d t,
\end{aligned}
$$

where

$$
C_{m-1-k}^{j}=\frac{(m-1-k) !}{j !(m-1-k-j) !}=\frac{\Gamma(m-k)}{\Gamma(j+1) \Gamma(m-k-j)} .
$$

Notice that, for $p>-\frac{q+1}{2}$ and $q>-1$,

$$
\begin{aligned}
\int_{0}^{\pi}(1+\cos t)^{p}(\sin t)^{q} d t & =2^{p+q} \int_{0}^{\pi}(\cos t / 2)^{2 p+q}(\sin t / 2)^{q} d t \\
& =2^{p+q} \mathbf{B}\left(p+\frac{q+1}{2}, \frac{q+1}{2}\right) \\
& =2^{p+q} \frac{\Gamma\left(p+\frac{q+1}{2}\right) \Gamma\left(\frac{q+1}{2}\right)}{\Gamma(p+q+1)} .
\end{aligned}
$$

We have, by (4.2)-(4.4),

$$
\begin{aligned}
& \left(k^{2}-(n-1)^{2} / 4-\Delta_{\mathbb{H}}\right)^{-1} \\
= & \frac{(2 \pi)^{-\frac{n}{2}} \Gamma(m+k)}{2^{k+\frac{1}{2}} \Gamma\left(k+\frac{1}{2}\right)}(\sinh \rho)^{1-2 m} . \\
& \sum_{j=0}^{m-1-k} \frac{\Gamma(m-k)}{\Gamma(j+1) \Gamma(m-k-j)}\left(\left(2 \sinh ^{2} \frac{\rho}{2}\right)^{j} 2^{m+k-1-j} \frac{\Gamma\left(m-j-\frac{1}{2}\right) \Gamma\left(k+\frac{1}{2}\right)}{\Gamma(m+k-j)}\right. \\
= & \frac{1}{4 \pi^{m+\frac{1}{2}}(\sinh \rho)^{2 m-1}} \sum_{j=0}^{m-k-1} \frac{\Gamma(m+k) \Gamma(m-k) \Gamma\left(m-j-\frac{1}{2}\right)}{\Gamma(j+1) \Gamma(m-k-j) \Gamma(m+k-j)}\left(\sinh \frac{\rho}{2}\right)^{2 j} .
\end{aligned}
$$

In general, we have the following: 
Lemma 4.2. Let $n=2 m+1$ and $l \in\{1,2, \cdots, m\}$. We have, for $k \in\{0,1, \cdots, m-l\}$ and $\rho>0$,

$$
\begin{aligned}
& {\left[\prod_{j=0}^{l-1}\left((k+j)^{2}-(n-1)^{2} / 4-\Delta_{\mathbb{H}}\right)\right]^{-1} } \\
= & \frac{1}{4 \Gamma(l) \pi^{m+\frac{1}{2}}(\sinh \rho)^{2 m-1}} \sum_{j=0}^{m-k-l} \frac{\Gamma(m+k) \Gamma(m-k-l+1) \Gamma\left(m-j-l+\frac{1}{2}\right)}{\Gamma(j+1) \Gamma(m-k-l-j+1) \Gamma(m+k-j)}\left(\sinh \frac{\rho}{2}\right)^{2 j+2 l-2} .
\end{aligned}
$$

Proof. We prove it by induction. By Lemma 4.1, (4.6) is valid for $l=1$. Assume (4.6) is valid for $l>1$. Then for $l+1$, we have

$$
\begin{aligned}
& {\left[\prod_{j=0}^{l}\left((k+j)^{2}-(n-1)^{2} / 4-\Delta_{\mathbb{H}}\right)\right]^{-1} } \\
= & \frac{1}{(2 k+l) l}\left\{\left[\prod_{j=0}^{l-1}\left((k+j)^{2}-(n-1)^{2} / 4-\Delta_{\mathbb{H}}\right)\right]^{-1}-\left[\prod_{j=1}^{l}\left((k+j)^{2}-(n-1)^{2} / 4-\Delta_{\mathbb{H}}\right)\right]^{-1}\right\} \\
= & \frac{1}{(2 k+l) l}\left\{\left[\prod_{j=0}^{l-1}\left((k+j)^{2}-(n-1)^{2} / 4-\Delta_{\mathbb{H}}\right)\right]^{-1}-\left[\prod_{j=0}^{l-1}\left((k+1+j)^{2}-(n-1)^{2} / 4-\Delta_{\mathbb{H}}\right)\right]^{-1}\right\} \\
= & \frac{\pi^{-m-\frac{1}{2}}(\sinh \rho)^{1-2 m}}{(2 k+l) l 4 \Gamma(l)}\left[\sum_{j=0}^{m-k-l} \frac{\Gamma(m+k) \Gamma(m-k-l+1) \Gamma\left(m-j-l+\frac{1}{2}\right)}{\Gamma(j+1) \Gamma(m-k-l-j+1) \Gamma(m+k-j)}\left(\sinh \frac{\rho}{2}\right)^{2 j+2 l-2}-\right. \\
& \left.\sum_{j=0}^{m-k-1-l} \frac{\Gamma(m+k+1) \Gamma(m-k-l) \Gamma\left(m-j-l+\frac{1}{2}\right)}{\Gamma(j+1) \Gamma(m-k-l-j) \Gamma(m+k+1-j)}\left(\sinh \frac{\rho}{2}\right)^{2 j+2 l-2}\right] .
\end{aligned}
$$

We compute

$$
\begin{aligned}
& \frac{\Gamma(m+k) \Gamma(m-k-l+1)}{\Gamma(m-k-l-j+1) \Gamma(m+k-j)}-\frac{\Gamma(m+k+1) \Gamma(m-k-l)}{\Gamma(m-k-l-j) \Gamma(m+k+1-j)} \\
= & \frac{\Gamma(m+k) \Gamma(m-k-l+1)}{\Gamma(m-k-l-j+1) \Gamma(m+k-j)}\left[1-\frac{(m+k)(m-k-l-j)}{(m+k-j)(m-k-l)}\right] \\
= & \frac{\Gamma(m+k) \Gamma(m-k-l+1)}{\Gamma(m-k-l-j+1) \Gamma(m+k-j)} \cdot \frac{(2 k+l) j}{(m+k-j)(m-k-l)} \\
= & (2 k+l) \frac{j \Gamma(m+k) \Gamma(m-k-l)}{\Gamma(m-k-l-j+1) \Gamma(m+k+1-j)} .
\end{aligned}
$$


Therefore, combining (4.7) and (4.8) yields

$$
\begin{aligned}
& {\left[\prod_{j=0}^{l}\left((k+j)^{2}-(n-1)^{2} / 4-\Delta_{\mathbb{H}}\right)\right]^{-1} } \\
= & \frac{\pi^{-m-\frac{1}{2}}(\sinh \rho)^{1-2 m}}{(2 k+l) l 4 \Gamma(l)}\left[\frac{\Gamma(m+k) \Gamma\left(k+\frac{1}{2}\right)}{\Gamma(2 k+l)}\left(\sinh \frac{\rho}{2}\right)^{2 m-2 k-2}+\right. \\
& \left.(2 k+l) \sum_{j=1}^{m-k-1-l} \frac{j \Gamma(m+k) \Gamma(m-k-l) \Gamma\left(m-j-l+\frac{1}{2}\right)}{\Gamma(j+1) \Gamma(m-k-l-j+1) \Gamma(m+k+1-j)}\left(\sinh \frac{\rho}{2}\right)^{2 j+2 l-2}\right] . \\
= & \frac{\pi^{-m-\frac{1}{2}}(\sinh \rho)^{1-2 m}}{4 \Gamma(l+1)}\left[\frac{\Gamma(m+k) \Gamma\left(k+\frac{1}{2}\right)}{\Gamma(2 k+l+1)}\left(\sinh \frac{\rho}{2}\right)^{2 m-2 k-2}+\right. \\
& \left.\sum_{j=0}^{m-k-2-l} \frac{\Gamma(m+k) \Gamma(m-k-l) \Gamma\left(m-j-l-\frac{1}{2}\right)}{\Gamma(j+1) \Gamma(m-k-l-j) \Gamma(m+k-j)}\left(\sinh \frac{\rho}{2}\right)^{2 j+2 l}\right] . \\
= & \frac{\pi^{-m-\frac{1}{2}}(\sinh \rho)^{1-2 m}}{4 \Gamma(l+1)} \sum_{j=0}^{m-k-1-l} \frac{\Gamma(m+k) \Gamma(m-k-l) \Gamma\left(m-j-l-\frac{1}{2}\right)}{\Gamma(j+1) \Gamma(m-k-l-j) \Gamma(m+k-j)}\left(\sinh \frac{\rho}{2}\right)^{2 j+2 l} .
\end{aligned}
$$

The desired result follows.

Before the proof of the next lemma, we recall the Hardy-Littlewood-Sobolev inequality on hyperbolic space which was first proved by Beckner [7] on upper half spaces (see also another proof in [43] for the equivalent form on hyperbolic balls).

Theorem 4.3. Let $0<\lambda<n$ and $p=\frac{2 n}{2 n-\lambda}$. Then for $f, g \in L^{p}\left(\mathbb{B}^{n}\right)$,

$$
\left|\int_{\mathbb{B}^{n}} \int_{\mathbb{B}^{n}} \frac{f(x) g(y)}{\left(2 \sinh \frac{\rho\left(T_{y}(x)\right)}{2}\right)^{\lambda}} d V_{x} d V_{y}\right| \leq C_{n, \lambda}\|f\|_{p}\|g\|_{p},
$$

where

$$
C_{n, \lambda}=\pi^{\lambda / 2} \frac{\Gamma(n / 2-\lambda / 2)}{\Gamma(n-\lambda / 2)}\left(\frac{\Gamma(n / 2)}{\Gamma(n)}\right)^{-1+\lambda / n}
$$

is the best constant for the classical Hardy-Littlewood-Sobolev constant on $\mathbb{R}^{n}$. Furthermore, the constant $C_{n, \lambda}$ is sharp for the inequality (4.10) and there is no nonzero extremal function for the inequality (4.10).

We are now ready to prove the following

Lemma 4.4. Let $n \geq 5$ be odd. There holds, for each $u \in C_{0}^{\infty}\left(\mathbb{B}^{n}\right)$,

$$
\int_{\mathbb{B}^{n}} \prod_{j=0}^{(n-3) / 2}\left(j^{2}-(n-1)^{2} / 4-\Delta_{\mathbb{H}}\right) u \cdot u d V \geq S_{n,(n-1) / 2}\left(\int_{\mathbb{B}^{n}}|u|^{2 n} d V\right)^{\frac{1}{n}} .
$$


Proof. It is sufficient to show the following inequality:

$$
\begin{aligned}
& \int_{\mathbb{B}^{n}} u(x)\left[\left(\prod_{j=0}^{(n-3) / 2}\left(j^{2}-(n-1)^{2} / 4-\Delta_{\mathbb{H}}\right)\right)^{-1} u\right](x) d V \\
\leq & \frac{C_{n, 1}}{\gamma_{n}(n-1)}\left(\int_{\mathbb{B}^{n}}|u(x)|^{\frac{2 n}{2 n-1}} d V\right)^{\frac{2 n-1}{n}} .
\end{aligned}
$$

Choosing $k=0$ and $l=m$ in Lemma 4.2, we have

$$
\begin{aligned}
{\left[\prod_{j=0}^{(n-3) / 2}\left(j^{2}-(n-1)^{2} / 4-\Delta_{\mathbb{H}}\right)\right]^{-1} } & =\frac{1}{4 \Gamma\left(\frac{n-1}{2}\right) \pi^{\frac{n}{2}}(\sinh \rho)^{n-2}} \Gamma\left(\frac{1}{2}\right)\left(\sinh \frac{\rho}{2}\right)^{n-3} \\
& =\frac{\Gamma\left(\frac{1}{2}\right)}{4 \Gamma\left(\frac{n-1}{2}\right) \pi^{\frac{n}{2}}} \cdot \frac{1}{2^{n-2}\left(\cosh \frac{\rho}{2}\right)^{n-2} \sinh \frac{\rho}{2}} \\
& =\frac{1}{\gamma_{n}(n-1)} \frac{1}{\left(\cosh \frac{\rho}{2}\right)^{n-2} 2 \sinh \frac{\rho}{2}} \\
& \leq \frac{1}{\gamma_{n}(n-1)} \cdot \frac{1}{2 \sinh \frac{\rho}{2}} .
\end{aligned}
$$

Therefore, by Theorem 4.3, we have

$$
\begin{aligned}
& \int_{\mathbb{B}^{n}} u(x)\left[\left(\prod_{k=0}^{(n-1) / 2}\left(k^{2}-(n-1)^{2} / 4-\Delta_{\mathbb{H}}\right)\right)^{-1} u\right](x) d V \\
\leq & \frac{1}{\gamma_{n}(n-1)} \int_{\mathbb{B}^{n}} \int_{\mathbb{B}^{n}} \frac{|u(x)| \cdot|u(y)|}{2 \sinh \frac{\rho\left(T_{y}(x)\right)}{2}} d V_{x} d V_{y} \\
\leq & \frac{C_{n, 1}}{\gamma_{n}(n-1)}\left(\int_{\mathbb{B}^{n}}|u(x)|^{\frac{2 n}{2 n-1}} d V\right)^{\frac{2 n-1}{n}} .
\end{aligned}
$$

This proves inequality (4.12). The proof of Lemma 4.4 is thus completed.

Proof of Theorem 1.6. By Lemma 4.4, it is enough to show

$$
\int_{\mathbb{B}^{n}}\left(P_{(n-1) / 2} u\right) u d V-\prod_{j=1}^{(n-1) / 2} \frac{(2 j-1)^{2}}{4} \int_{\mathbb{B}^{n}} u^{2} d V \geq \int_{\mathbb{B}^{n}} \prod_{j=0}^{(n-3) / 2}\left(j^{2}-(n-1)^{2} / 4-\Delta_{\mathbb{H}}\right) u \cdot u d V .
$$


By Plancherel's formula, we have

$$
\begin{aligned}
& \int_{\mathbb{B}^{n}}\left(P_{(n-1) / 2} u\right) u d V-\prod_{j=1}^{(n-1) / 2} \frac{(2 j-1)^{2}}{4} \int_{\mathbb{B}^{n}} u^{2} d V \\
= & D_{n} \int_{-\infty}^{+\infty} \int_{\mathbb{S}^{n-1}} \prod_{j=1}^{(n-1) / 2}\left(\frac{(n-1)^{2}+\lambda^{2}}{4}-\frac{(n+2 j-2)(n-2 j)}{4}\right)|\widehat{f}(\lambda, \zeta)|^{2}|\mathfrak{c}(\lambda)|^{-2} d \lambda d \sigma(\varsigma) \\
& -\prod_{j=1}^{(n-1) / 2} \frac{(2 j-1)^{2}}{4} \cdot D_{n} \int_{-\infty}^{+\infty} \int_{\mathbb{S}^{n-1}}|\widehat{f}(\lambda, \zeta)|^{2}|\mathfrak{c}(\lambda)|^{-2} d \lambda d \sigma(\varsigma) \\
= & D_{n} \int_{-\infty}^{+\infty} \int_{\mathbb{S}^{n-1}}\left(\prod_{j=1}^{(n-1) / 2} \frac{(2 j-1)^{2}+\lambda^{2}}{4}-\prod_{j=1}^{(n-1) / 2} \frac{(2 j-1)^{2}}{4}\right)|\widehat{f}(\lambda, \zeta)|^{2}|\mathfrak{c}(\lambda)|^{-2} d \lambda d \sigma(\varsigma) ;
\end{aligned}
$$

and

$$
\begin{aligned}
& \int_{\mathbb{B}^{n}} \prod_{j=0}^{(n-3) / 2}\left(j^{2}-(n-1)^{2} / 4-\Delta_{\mathbb{H}}\right) u \cdot u d V \\
= & D_{n} \int_{-\infty}^{+\infty} \int_{\mathbb{S}^{n-1}} \prod_{j=0}^{(n-3) / 2} \frac{j^{2}+\lambda^{2}}{4}|\widehat{f}(\lambda, \zeta)|^{2}|\mathfrak{c}(\lambda)|^{-2} d \lambda d \sigma(\varsigma) .
\end{aligned}
$$

Since

$$
\begin{aligned}
\prod_{j=1}^{(n-1) / 2} \frac{(2 j-1)^{2}+\lambda^{2}}{4}-\prod_{j=1}^{(n-1) / 2} \frac{(2 j-1)^{2}}{4} & \geq \frac{\lambda^{2}}{4} \prod_{j=2}^{(n-1) / 2} \frac{(2 j-1)^{2}+\lambda^{2}}{4} \\
& =\frac{\lambda^{2}}{4} \prod_{j=1}^{(n-3) / 2} \frac{(2 j+1)^{2}+\lambda^{2}}{4} \\
& \geq \frac{\lambda^{2}}{4} \prod_{j=1}^{(n-3) / 2} \frac{j^{2}+\lambda^{2}}{4} \\
& =\prod_{j=0}^{(n-3) / 2} \frac{j^{2}+\lambda^{2}}{4},
\end{aligned}
$$


we get, by (4.15) and (4.16),

$$
\begin{aligned}
& \int_{\mathbb{B}^{n}}\left(P_{(n-1) / 2} u\right) u d V-\prod_{j=1}^{(n-1) / 2} \frac{(2 j-1)^{2}}{4} \int_{\mathbb{B}^{n}} u^{2} d V \\
= & D_{n} \int_{-\infty}^{+\infty} \int_{\mathbb{S}^{n-1}}\left(\prod_{j=1}^{(n-1) / 2} \frac{(2 j-1)^{2}+\lambda^{2}}{4}-\prod_{j=1}^{(n-1) / 2} \frac{(2 j-1)^{2}}{4}\right)|\widehat{f}(\lambda, \zeta)|^{2}|\mathfrak{c}(\lambda)|^{-2} d \lambda d \sigma(\varsigma) \\
\geq & D_{n} \int_{-\infty}^{+\infty} \int_{\mathbb{S}^{n-1}} \prod_{j=0}^{(n-3) / 2} \frac{j^{2}+\lambda^{2}}{4}|\widehat{f}(\lambda, \zeta)|^{2}|\mathfrak{c}(\lambda)|^{-2} d \lambda d \sigma(\varsigma) \\
= & \int_{\mathbb{B}^{n}} \prod_{j=0}^{(n-3) / 2}\left(j^{2}-(n-1)^{2} / 4-\Delta_{\mathbb{H}}\right) u \cdot u d V .
\end{aligned}
$$

This proves the inequality (4.14). The proof of Theorem 1.6 is thereby completed.

5. Estimate of A LOWER BOUND FOR THE COEFFICIENT OF THE HARDY TERM IN THE Hardy-Sobolev-MaZ'ya IneQuality: Proof of Theorem 1.7

In this section, we will establish a lower bound for the coefficient $\lambda$ of the Hardy term in the higher order Hardy-Sobolev-Maz'ya inequality.

In what follows, $a=O(b)$ will stand for $a \leq C b$ and $a \sim b$ will stand for $C^{-1} b \leq a \leq C b$ with a positive constant $C$.

Set

$$
u_{k, \varepsilon}=\left(\frac{1-|x|^{2}}{2}\right)^{\frac{n-2 k}{2}}\left(\frac{2}{\varepsilon+|x|^{2}}\right)^{\frac{n-2 k}{2}} .
$$

Let $0<\delta<1$. For $|x|<\delta$, we have, by the Taylor series of $\left(\frac{1}{1-t}\right)^{\frac{n-2 k}{2}}$,

$$
\begin{aligned}
u_{k, \varepsilon} & =\left(\frac{1-|x|^{2}}{2}\right)^{\frac{n-2 k}{2}}\left(\frac{2}{\delta^{2}+\varepsilon}\right)^{\frac{n-2 k}{2}}\left(\frac{1}{1-\frac{\delta^{2}-|x|^{2}}{\delta^{2}+\varepsilon}}\right)^{\frac{n-2 k}{2}} \\
& =\left(\frac{1-|x|^{2}}{2}\right)^{\frac{n-2 k}{2}}\left(\frac{2}{\delta^{2}+\varepsilon}\right)^{\frac{n-2 k}{2}} \sum_{j=0}^{\infty} \frac{\Gamma\left(j+\frac{n-2 k}{2}\right)}{\Gamma(j+1) \Gamma\left(\frac{n-2 k}{2}\right)}\left(\frac{\delta^{2}-|x|^{2}}{\delta^{2}+\varepsilon}\right)^{j} .
\end{aligned}
$$

Set

$$
f_{k, \varepsilon}= \begin{cases}u_{k, \varepsilon}-\left(\frac{1-|x|^{2}}{2}\right)^{\frac{n-2 k}{2}}\left(\frac{2}{\delta^{2}+\varepsilon}\right)^{\frac{n-2 k}{2}} \sum_{j=0}^{k-1} \frac{\Gamma\left(j+\frac{n-2 k}{2}\right)}{\Gamma(j+1) \Gamma\left(\frac{n-2 k}{2}\right)}\left(\frac{1-|x|^{2}}{\delta^{2}+\varepsilon}\right)^{j}, & |x|<\delta \\ 0, & \delta \leq|x|<1 .\end{cases}
$$


It is known that

$$
(-\Delta)^{k}\left(\frac{2}{\varepsilon+|x|^{2}}\right)^{\frac{n-2 k}{2}}=\varepsilon^{k} \frac{\Gamma\left(\frac{n+2 k}{2}\right)}{\Gamma\left(\frac{n-2 k}{2}\right)}\left(\frac{2}{\varepsilon+|x|^{2}}\right)^{\frac{n+2 k}{2}}=\varepsilon^{k} \frac{S_{n, k}}{\omega_{n}^{2 k / n}}\left(\frac{2}{\varepsilon+|x|^{2}}\right)^{\frac{n+2 k}{2}}
$$

where $\omega_{n}$ is the surface area of $\mathbb{S}^{n}$ and $S_{n, k}=\frac{\Gamma\left(\frac{n+2 k}{2}\right)}{\Gamma\left(\frac{n-2 k}{2}\right)} \omega_{n}^{2 k / n}$ is the best Sobolev constant. Using the following identity (see [40], Theorem 2.3):

$$
\left(\frac{1-|x|^{2}}{2}\right)^{k+\frac{n}{2}}(-\Delta)^{k}\left[\left(\frac{1-|x|^{2}}{2}\right)^{k-\frac{n}{2}} f\right]=P_{k} f, \quad f \in C_{0}^{\infty}\left(\mathbb{B}^{n}\right), \quad k \in \mathbb{N},
$$

we have, for $|x|<\delta$,

$$
\begin{aligned}
& P_{k} u_{k, \varepsilon}=\varepsilon^{k} \frac{\Gamma\left(\frac{n+2 k}{2}\right)}{\Gamma\left(\frac{n-2 k}{2}\right)} u_{k, \varepsilon}^{\frac{n+2 k}{n-2 k}} ; \\
& P_{k} f_{k, \varepsilon}=P_{k} u_{k, \varepsilon}=\varepsilon^{k} \frac{\Gamma\left(\frac{n+2 k}{2}\right)}{\Gamma\left(\frac{n-2 k}{2}\right)} u_{k, \varepsilon}^{\frac{n+2 k}{n-2 k}} .
\end{aligned}
$$

The proof of Theorem 1.7 is divided into two parts.

5.1. Case I: $n \geq 4 k$.

Lemma 5.1. For $\varepsilon>0$ small enough, we have

$$
\begin{aligned}
\int_{\mathbb{B}^{n}}\left(P_{k} f_{k, \varepsilon}\right) f_{k, \varepsilon} d V & \leq \varepsilon^{k-n / 2} \frac{\Gamma\left(\frac{n+2 k}{2}\right)}{\Gamma\left(\frac{n-2 k}{2}\right)} \int_{\mathbb{R}^{n}}\left(\frac{2}{1+|x|^{2}}\right)^{n} d x ; \\
\int_{\mathbb{B}^{n}}\left|f_{k, \varepsilon}\right|^{\frac{2 n}{n-2 k}} d V & \geq \varepsilon^{-n / 2}\left[\int_{\mathbb{R}^{n}}\left(\frac{2}{1+|x|^{2}}\right)^{n} d x+O\left(\varepsilon^{\frac{n-2 k}{2}}\right)\right] \\
\int_{\mathbb{B}^{n}} f_{k, \varepsilon}^{2} d V & \sim \begin{cases}\varepsilon^{2 k-\frac{n}{2}}, & n>4 k ; \\
-\ln \varepsilon, & n=4 k .\end{cases}
\end{aligned}
$$

Proof. Set $B_{\delta}=\left\{x \in \mathbb{B}^{n}:|x|<\delta\right\}$. We have, by (5.3) and (5.6),

$$
\begin{aligned}
\int_{\mathbb{B}^{n}}\left(P_{k} f_{k, \varepsilon}\right) f_{k, \varepsilon} d V & =\varepsilon^{k} \frac{\Gamma\left(\frac{n+2 k}{2}\right)}{\Gamma\left(\frac{n-2 k}{2}\right)} \int_{B_{\delta}} u_{k, \varepsilon}^{\frac{n+2 k}{n-2 k}} f_{k, \varepsilon} d V \leq \varepsilon^{k} \frac{\Gamma\left(\frac{n+2 k}{2}\right)}{\Gamma\left(\frac{n-2 k}{2}\right)} \int_{B_{\delta}} u_{k, \varepsilon}^{\frac{2 n}{n-2 k}} d V \\
& =\varepsilon^{k} \frac{\Gamma\left(\frac{n+2 k}{2}\right)}{\Gamma\left(\frac{n-2 k}{2}\right)} \int_{B_{\delta}}\left(\frac{2}{\varepsilon+|x|^{2}}\right)^{n} d x \\
& =\varepsilon^{k-n / 2} \frac{\Gamma\left(\frac{n+2 k}{2}\right)}{\Gamma\left(\frac{n-2 k}{2}\right)} \int_{B_{\delta / \varepsilon}}\left(\frac{2}{1+|x|^{2}}\right)^{n} d x \\
& \leq \varepsilon^{k-n / 2} \frac{\Gamma\left(\frac{n+2 k}{2}\right)}{\Gamma\left(\frac{n-2 k}{2}\right)} \int_{\mathbb{R}^{n}}\left(\frac{2}{1+|x|^{2}}\right)^{n} d x .
\end{aligned}
$$

Next we prove the second inequality. Using the inequality

$$
(s-t)^{\alpha} \geq s^{\alpha}-\alpha s^{\alpha-1} t, \quad s \geq t \geq 0, \quad \alpha \geq 1,
$$


we have

$$
\begin{aligned}
& \int_{\mathbb{B}^{n}}\left|f_{k, \varepsilon}\right|^{\frac{2 n}{n-2 k}} d V \\
= & \int_{B_{\delta}}\left(u_{k, \varepsilon}-\left(\frac{1-|x|^{2}}{2}\right)^{\frac{n-2 k}{2}}\left(\frac{2}{\delta^{2}+\varepsilon}\right)^{\frac{n-2 k}{2}} \sum_{j=0}^{k-1} \frac{\Gamma\left(j+\frac{n-2 k}{2}\right)}{\Gamma(j+1) \Gamma\left(\frac{n-2 k}{2}\right)}\left(\frac{1-|x|^{2}}{\delta^{2}+\varepsilon}\right)^{j}\right)^{\frac{2 n}{n-2 k}} d V \\
\geq & \int_{B_{\delta}} u_{k, \varepsilon}^{\frac{2 n}{n-2 k}} d V-\frac{2 n}{n-2 k} \int_{B_{\delta}} u_{k, \varepsilon}^{\frac{n+2 k}{n-2 k}} \cdot \\
& \left(\frac{1-|x|^{2}}{2}\right)^{\frac{n-2 k}{2}}\left(\frac{2}{\delta^{2}+\varepsilon}\right)^{\frac{n-2 k}{2}} \sum_{j=0}^{k-1} \frac{\Gamma\left(j+\frac{n-2 k}{2}\right)}{\Gamma(j+1) \Gamma\left(\frac{n-2 k}{2}\right)}\left(\frac{1-|x|^{2}}{\delta^{2}+\varepsilon}\right)^{j} d V \\
= & \int_{B_{\delta}}\left(\frac{2 n}{\varepsilon+|x|^{2}}\right)^{n} d x-\frac{2 n}{n-2 k} \cdot \\
& \sum_{j=0} \frac{\Gamma\left(j+\frac{n-2 k}{2}\right)}{\Gamma(j+1) \Gamma\left(\frac{n-2 k}{2}\right)} \int_{B_{\delta}}\left(\frac{2}{\varepsilon+|x|^{2}}\right)^{\frac{n+2 k}{2}}\left(\frac{2}{\delta^{2}+\varepsilon}\right)^{\frac{n-2 k}{2}}\left(\frac{1-|x|^{2}}{\delta^{2}+\varepsilon}\right)^{j} d x .
\end{aligned}
$$

Easy computations lead to

$$
\begin{aligned}
& \int_{B_{\delta}}\left(\frac{2}{\varepsilon+|x|^{2}}\right)^{n}=\varepsilon^{-n / 2}\left[\int_{\mathbb{R}^{n}}\left(\frac{2}{1+|x|^{2}}\right)^{n} d x+O\left(\varepsilon^{\frac{n}{2}}\right)\right] ; \\
& \int_{B_{\delta}}\left(\frac{2}{\varepsilon+|x|^{2}}\right)^{\frac{n+2 k}{2}}\left(\frac{2}{\delta^{2}+\varepsilon}\right)^{\frac{n-2 k}{2}}\left(\frac{1-|x|^{2}}{\delta^{2}+\varepsilon}\right)^{j} d x=O\left(\varepsilon^{-k}\right), \quad j=0,1, \cdots, k-1 .
\end{aligned}
$$

Therefore, we have

$$
\int_{\mathbb{B}^{n}}\left|f_{k, \varepsilon}\right|^{\frac{2 n}{n-2 k}} d V=\varepsilon^{-n / 2}\left[\int_{\mathbb{R}^{n}}\left(\frac{2}{1+|x|^{2}}\right)^{n} d x+O\left(\varepsilon^{\frac{n-2 k}{2}}\right)\right]
$$

Finally, we prove the third inequality. We compute

$$
\begin{aligned}
\int_{\mathbb{B}^{n}} f_{k, \varepsilon}^{2} d V= & \int_{B_{\delta}} u_{k, \varepsilon}^{2} d V-2 \int_{B_{\delta}} u_{k, \varepsilon}\left(\frac{1-|x|^{2}}{2}\right)^{\frac{n-2 k}{2}} . \\
& \left(\frac{2}{\delta^{2}+\varepsilon}\right)^{\frac{n-2 k}{2}} \sum_{j=0}^{k-1} \frac{\Gamma\left(j+\frac{n-2 k}{2}\right)}{\Gamma(j+1) \Gamma\left(\frac{n-2 k}{2}\right)}\left(\frac{1-|x|^{2}}{\delta^{2}+\varepsilon}\right)^{j} d V+ \\
& \int_{B_{\delta}}\left|\left(\frac{1-|x|^{2}}{2}\right)^{\frac{n-2 k}{2}}\left(\frac{2}{\delta^{2}+\varepsilon}\right)^{\frac{n-2 k}{2}} \sum_{j=0}^{k-1} \frac{\Gamma\left(j+\frac{n-2 k}{2}\right)}{\Gamma(j+1) \Gamma\left(\frac{n-2 k}{2}\right)}\left(\frac{1-|x|^{2}}{\delta^{2}+\varepsilon}\right)^{j}\right|^{2} d V .
\end{aligned}
$$


Simple calculations lead to

$$
\begin{aligned}
& \int_{\mathbb{B}^{n}} u_{k, \varepsilon}^{2} d V=\int_{B_{\delta}}\left(\frac{2}{\varepsilon+|x|^{2}}\right)^{n-2 k}\left(\frac{2}{1-|x|^{2}}\right)^{2 k} d x \\
& \sim\left\{\begin{array}{l}
\varepsilon^{2 k-\frac{n}{2}}, \quad n>4 k ; \\
-\ln \varepsilon, \quad n=4 k,
\end{array}\right. \\
& \int_{B_{\delta}} u_{k, \varepsilon}\left(\frac{1-|x|^{2}}{2}\right)^{\frac{n-2 k}{2}}\left(\frac{2}{\delta^{2}+\varepsilon}\right)^{\frac{n-2 k}{2}}\left(\frac{1-|x|^{2}}{\delta^{2}+\varepsilon}\right)^{j} d V=O\left(\varepsilon^{k}\right), \quad k=0,1, \cdots, k-1, \\
& \int_{B_{\delta}}\left|\left(\frac{1-|x|^{2}}{2}\right)^{\frac{n-2 k}{2}}\left(\frac{2}{\delta^{2}+\varepsilon}\right)^{\frac{n-2 k}{2}} \sum_{j=0}^{k-1} \frac{\Gamma\left(j+\frac{n-2 k}{2}\right)}{\Gamma(j+1) \Gamma\left(\frac{n-2 k}{2}\right)}\left(\frac{1-|x|^{2}}{\delta^{2}+\varepsilon}\right)^{j}\right|^{2} d V \sim 1 .
\end{aligned}
$$

Therefore, we have, by (5.9),

$$
\int_{\mathbb{B}^{n}} f_{k, \varepsilon}^{2} d V \sim \begin{cases}\varepsilon^{2 k-\frac{n}{2}}, & n>4 k \\ -\ln \varepsilon, & n=4 k\end{cases}
$$

Lemma 5.2. Let $2 \leq k \leq \frac{n}{4}$. Suppose that there exists $\lambda \in \mathbb{R}$ such that for any $u \in C_{0}^{\infty}\left(\mathbb{B}^{n}\right)$,

$$
\int_{\mathbb{B}^{n}}\left(P_{k} u\right) u d V+\lambda \int_{\mathbb{B}^{n}} u^{2} d V \geq S_{n, k}\left(\int_{\mathbb{B}^{n}}|u|^{\frac{2 n}{n-2 k}} d V\right)^{\frac{n-2 k}{n}},
$$

where $S_{n, k}$ is the best $k$-th order Sobolev constant. Then $\lambda \geq 0$.

Proof. By Lemma 5.1, if $n>4 k$, then for $\varepsilon>0$ small enough, we have, for some $C_{1}>0$,

$$
\begin{aligned}
& \frac{\int_{\mathbb{B}^{n}} P_{k} f_{k, \varepsilon} f_{k, \varepsilon} d V+\lambda \int_{\mathbb{B}^{n}} f_{k, \varepsilon}^{2} d V}{\left(\int_{\mathbb{B}^{n}}\left|f_{k, \varepsilon}\right|^{\frac{2 n}{n-2 k}} d V\right)^{\frac{n-2 k}{n}}} \leq \frac{\frac{\Gamma\left(\frac{n+2 k}{2}\right)}{\Gamma\left(\frac{n-2 k}{2}\right)} \int_{\mathbb{R}^{n}}\left(\frac{2}{1+|x|^{2}}\right)^{n} d x+C_{1} \lambda \varepsilon^{k}}{\left[\int_{\mathbb{R}^{n}}\left(\frac{2}{1+|x|^{2}}\right)^{n} d x+O\left(\varepsilon^{\frac{n-2 k}{2}}\right)\right]^{\frac{n-2 k}{n}}} \\
= & \frac{\Gamma\left(\frac{n+2 k}{2}\right)}{\Gamma\left(\frac{n-2 k}{2}\right)}\left[\int_{\mathbb{R}^{n}}\left(\frac{2}{1+|x|^{2}}\right)^{n} d x\right]^{\frac{2 k}{n}}+O\left(\varepsilon^{\frac{n-2 k}{2}}\right)+\frac{C_{1} \lambda \varepsilon^{k}}{\left[\int_{\mathbb{R}^{n}}\left(\frac{2}{1+|x|^{2}}\right)^{n} d x\right]^{\frac{n-2 k}{n}}}+O\left(\varepsilon^{\frac{n}{2}}\right) \\
= & S_{n, k}+\frac{C_{1} \lambda \varepsilon^{k}}{\left[\int_{\mathbb{R}^{n}}\left(\frac{2}{1+|x|^{2}}\right)^{n} d x\right]^{\frac{n-2 k}{n}}}+O\left(\varepsilon^{\frac{n-2 k}{2}}\right) .
\end{aligned}
$$

To get the last equality, we use the fact

$$
\int_{\mathbb{R}^{n}}\left(\frac{2}{1+|x|^{2}}\right)^{n} d x=2^{n} \omega_{n-1} \int_{0}^{\infty} \frac{r^{n-1}}{\left(1+r^{2}\right)^{n}} d r=2^{n-1} \omega_{n-1} \int_{0}^{\infty} \frac{t^{\frac{n}{2}-1}}{(1+t)^{n}} d t=\omega_{n}
$$


Since (5.10) implies

$$
\frac{\int_{\mathbb{B}^{n}} P_{k} f_{k, \varepsilon} f_{k, \varepsilon} d V+\lambda \int_{\mathbb{B}^{n}} f_{k, \varepsilon}^{2} d V}{\left(\int_{\mathbb{B}^{n}}\left|f_{k, \varepsilon}\right|^{\frac{2 n}{n-2 k}} d V\right)^{\frac{n-2 k}{n}}} \geq S_{n, k}
$$

we have, by (5.11), $\lambda \geq 0$.

Similarly, in the case $n=4 k$, we have, for some $C_{2}>0$,

$$
\begin{aligned}
S_{n, k} & \leq \frac{\int_{\mathbb{B}^{n}} P_{k} f_{k, \varepsilon} f_{k, \varepsilon} d V+\lambda \int_{\mathbb{B}^{n}} f_{k, \varepsilon}^{2} d V}{\left(\int_{\mathbb{B}^{n}}\left|f_{k, \varepsilon}\right|^{\frac{2 n}{n-2 k}} d V\right)^{\frac{n-2 k}{n}}} \leq \frac{\frac{\Gamma\left(\frac{n+2 k}{2}\right)}{\Gamma\left(\frac{n-2 k}{2}\right)} \int_{\mathbb{R}^{n}}\left(\frac{2}{1+|x|^{2}}\right)^{n} d x-C_{2} \lambda \varepsilon^{k} \ln \varepsilon}{\left[\int_{\mathbb{R}^{n}}\left(\frac{2}{1+|x|^{2}}\right)^{n} d x+O\left(\varepsilon^{k}\right)\right]^{\frac{n-2 k}{n}}} \\
& \leq \frac{\Gamma\left(\frac{n+2 k}{2}\right)}{\Gamma\left(\frac{n-2 k}{2}\right)}\left[\int_{\mathbb{R}^{n}}\left(\frac{2}{1+|x|^{2}}\right)^{n} d x\right]^{\frac{2 k}{n}}+O\left(\varepsilon^{k}\right)-\frac{C_{2} \lambda \varepsilon^{k} \ln \varepsilon}{\left[\int_{\mathbb{R}^{n}}\left(\frac{2}{1+|x|^{2}}\right)^{n} d x\right]^{\frac{n-2 k}{n}}+O\left(\varepsilon^{2 k} \ln \varepsilon\right)} \\
& =S_{n, k}-\frac{C_{2} \lambda \varepsilon^{k} \ln \varepsilon}{\left[\int_{\mathbb{R}^{n}}\left(\frac{2}{1+|x|^{2}}\right)^{n} d x\right]^{\frac{n-2 k}{n}}}+O\left(\varepsilon^{k}\right) .
\end{aligned}
$$

Therefore, we must also have $\lambda \geq 0$.

5.2. Case II: $2 k+2 \leq n<4 k$. We choose $\delta=1$ in (5.2) and set

$$
g_{k, \varepsilon}=u_{k, \varepsilon}-\left(\frac{1-|x|^{2}}{2}\right)^{\frac{n-2 k}{2}}\left(\frac{2}{1+\varepsilon}\right)^{\frac{n-2 k}{2}} \sum_{j=0}^{k-1} \frac{\Gamma\left(j+\frac{n-2 k}{2}\right)}{\Gamma(j+1) \Gamma\left(\frac{n-2 k}{2}\right)}\left(\frac{1-|x|^{2}}{1+\varepsilon}\right)^{j} .
$$

Then

$$
P_{k} g_{k, \varepsilon}=P_{k} u_{k, \varepsilon}=\varepsilon^{k} \frac{\Gamma\left(\frac{n+2 k}{2}\right)}{\Gamma\left(\frac{n-2 k}{2}\right)} u_{k, \varepsilon}^{\frac{n+2 k}{n-2 k}}
$$

and

$$
\begin{aligned}
\int_{\mathbb{B}^{n}}\left(P_{k} g_{k, \varepsilon}\right) g_{k, \varepsilon} d V & \leq \varepsilon^{k} \frac{\Gamma\left(\frac{n+2 k}{2}\right)}{\Gamma\left(\frac{n-2 k}{2}\right)} \int_{B_{\delta}} u_{k, \varepsilon}^{\frac{2 n}{n-2 k}} d V \\
& =\varepsilon^{k} \frac{\Gamma\left(\frac{n+2 k}{2}\right)}{\Gamma\left(\frac{n-2 k}{2}\right)} \int_{B_{\delta}}\left(\frac{2}{\varepsilon+|x|^{2}}\right)^{n} d x \\
& \leq \varepsilon^{k-n / 2} \frac{\Gamma\left(\frac{n+2 k}{2}\right)}{\Gamma\left(\frac{n-2 k}{2}\right)} \int_{\mathbb{R}^{n}}\left(\frac{2}{1+|x|^{2}}\right)^{n} d x
\end{aligned}
$$


Easy calculations lead to

$$
\begin{aligned}
& \left.\int_{\mathbb{B}^{n}} \mid g_{k, \varepsilon}\right)\left.\right|^{2} d V \\
= & \int_{\mathbb{B}^{n}}\left|\left(\frac{2}{\varepsilon+|x|^{2}}\right)^{\frac{n-2 k}{2}}-\left(\frac{2}{1+\varepsilon}\right)^{\frac{n-2 k}{2}} \sum_{j=0}^{k-1} \frac{\Gamma\left(j+\frac{n-2 k}{2}\right)}{\Gamma(j+1) \Gamma\left(\frac{n-2 k}{2}\right)}\left(\frac{1-|x|^{2}}{1+\varepsilon}\right)^{j}\right|^{2} \frac{2^{2 k}}{\left(1-|x|^{2}\right)^{2 k}} d x \\
= & 2^{n} \int_{\mathbb{B}^{n}}\left|\frac{1}{|x|^{n-2 k}}-\sum_{j=0}^{k-1} \frac{\Gamma\left(j+\frac{n-2 k}{2}\right)}{\Gamma(j+1) \Gamma\left(\frac{n-2 k}{2}\right)}\left(1-|x|^{2}\right)^{j}\right|^{2} \frac{1}{\left(1-|x|^{2}\right)^{2 k}} d x+o(1) .
\end{aligned}
$$

Lemma 5.3. For $\varepsilon>0$ small enough, we have

$$
\begin{aligned}
\left(\int_{\mathbb{B}^{n}}\left|g_{k, \varepsilon}\right|^{\frac{2 n}{n-2 k}} d x\right)^{\frac{n-2 k}{n}} & \geq \varepsilon^{k-\frac{n}{2}}\left(\int_{\mathbb{R}^{n}} \frac{1}{\left(1+|x|^{2}\right)^{n}} d x\right)^{\frac{n-2 k}{n}} \cdot \\
& {\left[1-\varepsilon^{\frac{n-2 k}{2}} 2^{\frac{n}{2}-k+1} \sum_{j=0}^{k-1} \frac{\Gamma\left(j+\frac{n-2 k}{2}\right)}{\Gamma(j+1) \Gamma\left(\frac{n-2 k}{2}\right)} \frac{\int_{\mathbb{R}^{n}} \frac{1}{\left(1+|x|^{2}\right)^{\frac{n+2 k}{2}}} d x}{\int_{\mathbb{R}^{n}} \frac{1}{\left(1+|x|^{2}\right)^{n}} d x}+O\left(\varepsilon^{\frac{n}{2}}\right)\right] . }
\end{aligned}
$$

Proof. We have, for $\varepsilon>0$ small enough,

$$
\begin{aligned}
& \int_{\mathbb{B}^{n}}\left|g_{k, \varepsilon}\right|^{\frac{2 n}{n-2 k}} d x \\
= & \int_{\mathbb{B}^{n}}\left|u_{k, \varepsilon}-\left(\frac{2}{1+\varepsilon}\right)^{\frac{n-2 k}{2}} \sum_{j=0}^{k-1} \frac{\Gamma\left(j+\frac{n-2 k}{2}\right)}{\Gamma(j+1) \Gamma\left(\frac{n-2 k}{2}\right)}\left(\frac{1-|x|^{2}}{1+\varepsilon}\right)^{j}\right|^{\frac{2 n}{n-2 k}} d x \\
= & \int_{\mathbb{B}^{n}}\left|\left(\frac{1}{\varepsilon+|x|^{2}}\right)^{\frac{n-2 k}{2}}-\left(\frac{2}{1+\varepsilon}\right)^{\frac{n-2 k}{2}} \sum_{j=0}^{k-1} \frac{\Gamma\left(j+\frac{n-2 k}{2}\right)}{\Gamma(j+1) \Gamma\left(\frac{n-2 k}{2}\right)}\left(\frac{1-|x|^{2}}{1+\varepsilon}\right)^{j}\right|^{\frac{2 n}{n-2 k}} d x \\
\geq & \int_{\mathbb{B}^{n}}\left(\frac{1}{\varepsilon+|x|^{2}}\right)^{n} d x- \\
& \frac{2 n}{n-2 k}\left(\frac{2}{1+\varepsilon}\right)^{\frac{n-2 k}{2}} \sum_{j=0}^{k-1} \frac{\Gamma\left(j+\frac{n-2 k}{2}\right)}{\Gamma(j+1) \Gamma\left(\frac{n-2 k}{2}\right)} \int_{\mathbb{B}^{n}}\left(\frac{1}{\varepsilon+|x|^{2}}\right)^{\frac{n+2 k}{2}}\left(\frac{1-|x|^{2}}{1+\varepsilon}\right)^{j} d x \\
\geq & \int_{\mathbb{B}^{n}}\left(\frac{1}{\varepsilon+|x|^{2}}\right)^{n} d x-\frac{2^{\frac{n}{2}-k+1}}{n-2 k} \sum_{j=0}^{k-1} \frac{\Gamma\left(j+\frac{n-2 k}{2}\right)}{\Gamma(j+1) \Gamma\left(\frac{n-2 k}{2}\right)} \int_{\mathbb{B}^{n}}\left(\frac{1}{\varepsilon+|x|^{2}}\right)^{\frac{n+2 k}{2}} d x .
\end{aligned}
$$


An easy computation leads to

$$
\begin{aligned}
\int_{\mathbb{B}^{n}}\left(\frac{1}{\varepsilon+|x|^{2}}\right)^{n} d x & =\varepsilon^{-\frac{n}{2}}\left[\int_{\mathbb{R}^{n}}\left(\frac{2}{1+|x|^{2}}\right)^{n} d x+O\left(\varepsilon^{\frac{n}{2}}\right)\right] \\
\int_{\mathbb{B}^{n}}\left(\frac{1}{\varepsilon+|x|^{2}}\right)^{\frac{n+2 k}{2}} d x & =\varepsilon^{-k}\left[\int_{\mathbb{R}^{n}}\left(\frac{1}{1+|x|^{2}}\right)^{\frac{n+2 k}{2}} d x+O\left(\varepsilon^{k}\right)\right] d x .
\end{aligned}
$$

Therefore, we have, by (5.16),

$$
\begin{aligned}
\int_{\mathbb{B}^{n}}\left|g_{k, \varepsilon}\right|^{\frac{2 n}{n-2 k}} d x & \geq \varepsilon^{-\frac{n}{2}} \int_{\mathbb{R}^{n}} \frac{1}{\left(1+|x|^{2}\right)^{n}} d x . \\
& {\left[1-\frac{\varepsilon^{\frac{n-2 k}{2}} 2^{\frac{n}{2}-k+1} n}{n-2 k} \sum_{j=0}^{k-1} \frac{\Gamma\left(j+\frac{n-2 k}{2}\right)}{\Gamma(j+1) \Gamma\left(\frac{n-2 k}{2}\right)} \frac{\int_{\mathbb{R}^{n}} \frac{1}{\left(1+|x|^{2}\right)^{\frac{n+2 k}{2}}} d x}{\int_{\mathbb{R}^{n}} \frac{1}{\left(1+|x|^{2}\right)^{n}} d x}+O\left(\varepsilon^{\frac{n}{2}}\right)\right] . }
\end{aligned}
$$

The desired result follows.

Lemma 5.4. Let $k \geq 2$ and $2 k+2 \leq n \leq 4 k-1$. Suppose that there exists $\lambda \in \mathbb{R}$ such that for any $u \in C_{0}^{\infty}\left(\mathbb{B}^{n}\right)$,

$$
\int_{\mathbb{B}^{n}}\left(P_{k} u\right) u d V+\lambda \int_{\mathbb{B}^{n}} u^{2} d V \geq S_{n, k}\left(\int_{\mathbb{B}^{n}}|u|^{\frac{2 n}{n-2 k}} d V\right)^{\frac{n-2 k}{n}},
$$

where $S_{n, k}$ is the best $k$-th order Sobolev constant. Then

$$
\lambda \geq-\frac{\Gamma(n / 2) \Gamma(k)}{2^{\frac{n+2 k}{2}} \Gamma\left(\frac{n-2 k}{2}\right) \int_{0}^{1}\left[r^{2 k-n}-\sum_{j=0}^{k-1} \frac{\Gamma\left(j+\frac{n-2 k}{2}\right)}{\Gamma(j+1) \Gamma\left(\frac{n-2 k}{2}\right)}\left(1-r^{2}\right)^{j}\right]^{2} \frac{r^{n-1}}{\left(1-r^{2}\right)^{2 k}} d r} \sum_{j=0}^{k-1} \frac{\Gamma\left(j+\frac{n-2 k}{2}\right)}{\Gamma(j+1) \Gamma\left(\frac{n-2 k}{2}\right)} .
$$

Proof. We have, by Lemma 5.3,

$$
\begin{aligned}
S_{n, k} & \leq \frac{\int_{\mathbb{B}^{n}} P_{k} g_{k, \varepsilon} g_{k, \varepsilon} d V+\lambda \int_{\mathbb{B}^{n}} g_{k, \varepsilon}^{2} d V}{\left(\int_{\mathbb{B}^{n}}\left|g_{k, \varepsilon}\right|^{\frac{2 n}{n-2 k}} d V\right)^{\frac{n-2 k}{n}}} \\
& \leq S_{n, k} \frac{1+\varepsilon^{\frac{n}{2}-k} \lambda \frac{\Gamma\left(\frac{n-2 k}{2}\right)}{\Gamma\left(\frac{n+2 k}{2}\right)} \frac{2^{n} \int_{\mathbb{B}^{n}}\left|\frac{1}{|x|^{n-2 k}}-\sum_{j=0}^{k-1} \frac{\Gamma\left(j+\frac{n-2 k}{2}\right)}{\Gamma(j+1) \Gamma\left(\frac{n-2 k}{2}\right)}\left(1-|x|^{2}\right)^{j}\right|^{2} \frac{1}{\left(1-|x|^{2}\right)^{2 k}} d x}{\int_{\mathbb{R}^{n}} \frac{1}{\left(1+|x|^{2}\right)^{n}} d x}+O\left(\varepsilon^{\frac{n}{2}-k}\right)}{1-\varepsilon^{\frac{n}{2}-k} 2^{\frac{n}{2}-k+1} \sum_{j=0}^{k-1} \frac{\Gamma\left(j+\frac{n-2 k}{2}\right)}{\Gamma(j+1) \Gamma\left(\frac{n-2 k}{2}\right)} \frac{\int_{\mathbb{R}^{n}} \frac{1}{\left(1+|x|^{2}\right)^{\frac{n+2 k}{2}}} d x}{\int_{\mathbb{R}^{n}} \frac{1}{\left(1+|x|^{2}\right)^{n}} d x}+O\left(\varepsilon^{\frac{n}{2}}\right)} .
\end{aligned}
$$


Therefore, we must have

$$
\begin{aligned}
\lambda \geq-\frac{2^{\frac{n}{2}-k+1} \frac{\Gamma\left(\frac{n+2 k}{2}\right)}{\Gamma\left(\frac{n-2 k}{2}\right)} \sum_{j=0}^{k-1} \frac{\Gamma\left(j+\frac{n-2 k}{2}\right)}{\Gamma(j+1) \Gamma\left(\frac{n-2 k}{2}\right)} \int_{\mathbb{R}^{n}} \frac{1}{\left(1+|x|^{2}\right)^{\frac{n+2 k}{2}}} d x}{2^{n} \int_{\mathbb{B}^{n}} \mid \frac{1}{|x|^{n-2 k}}-\sum_{j=0}^{k-1} \frac{\Gamma\left(j+\frac{n-2 k}{2}\right)}{\Gamma(j+1) \Gamma\left(\frac{n-2 k}{2}\right)}\left(1-\left.\left.|x|^{2}\right|^{j}\right|^{2} \frac{1}{\left(1-|x|^{2}\right)^{2 k}} d x\right.} \\
=-\frac{\frac{\Gamma\left(\frac{n+2 k}{2}\right)}{\Gamma\left(\frac{n-2 k}{2}\right)} \sum_{j=0}^{k-1} \frac{\Gamma\left(j+\frac{n-2 k}{2}\right)}{\Gamma(j+1) \Gamma\left(\frac{n-2 k}{2}\right)} \int_{0}^{\infty} \frac{r^{n-1}}{\left(1+r^{2}\right)^{\frac{n+2 k}{2}}} d r}{2^{\frac{n}{2}+k-1} \int_{0}^{\infty}\left|\frac{1}{r^{n-2 k}}-\sum_{j=0}^{k-1} \frac{\Gamma\left(j+\frac{n-2 k}{2}\right)}{\Gamma(j+1) \Gamma\left(\frac{n-2 k}{2}\right)}\left(1-r^{2}\right)^{j}\right|^{2} \frac{r^{n-1} d r}{\left(1-r^{2}\right)^{2 k}}} \\
2^{\frac{n+2 k}{2} \Gamma\left(\frac{n-2 k}{2}\right) \int_{0}^{1}\left[r^{2 k-n}-\sum_{j=0}^{k-1} \frac{\Gamma\left(j+\frac{n-2 k}{2}\right)}{\Gamma(j+1) \Gamma\left(\frac{n-2 k}{2}\right)}\left(1-r^{2}\right)^{j}\right]^{2} \frac{r^{n-1}}{\left(1-r^{2}\right)^{2 k}} d r} \sum_{j=0}^{k-1} \frac{\Gamma\left(j+\frac{n-2 k}{2}\right)}{\Gamma(j+1) \Gamma\left(\frac{n-2 k}{2}\right)} .
\end{aligned}
$$

To get the last equality, we use the fact

$$
\int_{0}^{\infty} \frac{r^{n-1}}{\left(1+r^{2}\right)^{\frac{n+2 k}{2}}} d r=\frac{1}{2} \int_{0}^{\infty} \frac{t^{\frac{n}{2}-1}}{(1+t)^{\frac{n+2 k}{2}}} d t=\frac{1}{2} \mathbf{B}\left(\frac{n}{2}, k\right)=\frac{1}{2} \frac{\Gamma(n / 2) \Gamma(k)}{\Gamma(k+n / 2)} .
$$

This completes the proof of Lemma 5.4.

Proof of Theorem 1.7 Combining Lemma 5.2 and 5.4, Theorem 1.7 follows.

6. Precise expression and optimal bound for Green's function of Paneitz AND GJMS operators: Proof of Theorems 1.10

In this section, we will provide a proof of Theorems 1.10. Namely, we will provide a precise expression and optimal bound for Green's function of Paneitz and GJMS operators $P_{k}$.

The proofs are divided into two parts.

\subsection{Case 1: $n$ is even.}

Lemma 6.1. If $n=2 m$ and $0 \leq k \leq m-1$, then

$$
\begin{aligned}
& \left((k+1 / 2)^{2}-(n-1)^{2} / 4-\Delta_{\mathbb{H}}\right)^{-1} \\
= & \frac{1}{4 \pi^{m+\frac{1}{2}}(\sinh \rho)^{2 m-1}} \sum_{j=0}^{m-k-1} \frac{\Gamma(m+k) \Gamma(m-k) \Gamma\left(m-j-\frac{1}{2}\right)}{\Gamma(j+1) \Gamma(m-k-j) \Gamma(m+k-j)}\left(\sinh \frac{\rho}{2}\right)^{2 j} .
\end{aligned}
$$


Proof. If $n=2 m$ and $0 \leq k \leq m-2$, then by (3.12) and the binomial theorem, we have

$$
\begin{aligned}
& \left((k+1 / 2)^{2}-(n-1)^{2} / 4-\Delta_{\mathbb{H}}\right)^{-1} \\
= & \frac{(2 \pi)^{-\frac{n}{2}} \Gamma(m+k)}{2^{k+1} \Gamma(k+1)}(\sinh \rho)^{2-2 m} \int_{0}^{\pi}(\cosh \rho+\cos t)^{m-2-k}(\sin t)^{2 k+1} d t \\
= & \frac{(2 \pi)^{-\frac{n}{2}} \Gamma(m+k)}{2^{k+1} \Gamma(k+1)}(\sinh \rho)^{2-2 m} \int_{0}^{\pi}\left(2 \sinh ^{2} \frac{\rho}{2}+1+\cos t\right)^{m-2-k}(\sin t)^{2 k+1} d t \\
= & \frac{(2 \pi)^{-\frac{n}{2}} \Gamma(m+k)}{2^{k+1} \Gamma(k+1)}(\sinh \rho)^{2-2 m} \sum_{j=0}^{m-2-k} C_{m-2-k}^{j}\left(2 \sinh ^{2} \frac{\rho}{2}\right)^{j} \int_{0}^{\pi}(1+\cos t)^{m-2-k-j}(\sin t)^{2 k+1} d t .
\end{aligned}
$$

We have, by (6.2) and (4.4),

$$
\begin{aligned}
& \left((k+1 / 2)^{2}-(n-1)^{2} / 4-\Delta_{\mathbb{H}}\right)^{-1} \\
= & \frac{(2 \pi)^{-\frac{n}{2}} \Gamma(m+k)}{2^{k+1} \Gamma(k+1)}(\sinh \rho)^{2-2 m} . \\
& \sum_{j=0}^{m-2-k} \frac{\Gamma(m-1-k)}{\Gamma(j+1) \Gamma(m-1-k-j)}\left(2 \sinh ^{2} \frac{\rho}{2}\right)^{j} 2^{m+k-1-j} \frac{\Gamma(m-j-1) \Gamma(k+1)}{\Gamma(m+k-j)} \\
= & \frac{1}{4 \pi^{m}(\sinh \rho)^{2 m-2}} \sum_{j=0}^{m-k-2} \frac{\Gamma(m+k) \Gamma(m-k-1) \Gamma(m-j-1)}{\Gamma(j+1) \Gamma(m-1-k-j) \Gamma(m+k-j)}\left(\sinh \frac{\rho}{2}\right)^{2 j} .
\end{aligned}
$$

In general, we have the following:

Lemma 6.2. Let $n=2 m$ and $l \in\{1,2, \cdots, m-1\}$. We have, for $k \in\{0,1, \cdots, m-1-l\}$ and $\rho>0$,

$$
\begin{aligned}
& {\left[\prod_{j=0}^{l-1}\left((k+j+1 / 2)^{2}-(n-1)^{2} / 4-\Delta_{\mathbb{H}}\right)\right]^{-1} } \\
= & \frac{1}{4 \Gamma(l) \pi^{m}(\sinh \rho)^{2 m-2}} \sum_{j=0}^{m-1-k-l} \frac{\Gamma(m+k) \Gamma(m-k-l) \Gamma(m-j-l)}{\Gamma(j+1) \Gamma(m-k-l-j) \Gamma(m+k-j)}\left(\sinh \frac{\rho}{2}\right)^{2 j+2 l-2}
\end{aligned}
$$


Proof. We prove it by induction. By Lemma 6.1, (6.4) is valid for $l=1$. Assume (6.4) is valid for $l>1$. Then for $l+1$, we have

$$
\begin{aligned}
& {\left[\prod_{j=0}^{l}\left((k+j+1 / 2)^{2}-(n-1)^{2} / 4-\Delta_{\mathbb{H}}\right)\right]^{-1}} \\
& =\frac{1}{(2 k+1+l) l}\left\{\left[\prod_{j=0}^{l-1}\left((k+j+1 / 2)^{2}-(n-1)^{2} / 4-\Delta_{\mathbb{H}}\right)\right]^{-1}-\right. \\
& \left.\left[\prod_{j=1}^{l}\left((k+j+1 / 2)^{2}-(n-1)^{2} / 4-\Delta_{\mathbb{H}}\right)\right]^{-1}\right\} \\
& (6.5)=\frac{1}{(2 k+1+l) l}\left\{\left[\prod_{j=0}^{l-1}\left((k+j+1 / 2)^{2}-(n-1)^{2} / 4-\Delta_{\mathbb{H}}\right)\right]^{-1}-\right. \\
& \left.\left[\prod_{j=0}^{l-1}\left((k+j+1+1 / 2)^{2}-(n-1)^{2} / 4-\Delta_{\mathbb{H}}\right)\right]^{-1}\right\} \\
& =\frac{\pi^{-m}(\sinh \rho)^{2-2 m}}{(2 k+1+l) l 4 \Gamma(l)}\left[\sum_{j=0}^{m-1-k-l} \frac{\Gamma(m+k) \Gamma(m-k-l) \Gamma(m-j-l)}{\Gamma(j+1) \Gamma(m-k-l-j) \Gamma(m+k-j)}\left(\sinh \frac{\rho}{2}\right)^{2 j+2 l-2}\right. \\
& \left.-\sum_{j=0}^{m-2-k-l} \frac{\Gamma(m+k+1) \Gamma(m-1-k-l) \Gamma(m-j-l)}{\Gamma(j+1) \Gamma(m-1-k-l-j) \Gamma(m+k+1-j)}\left(\sinh \frac{\rho}{2}\right)^{2 j+2 l-2}\right] .
\end{aligned}
$$

We compute

$$
\begin{aligned}
& \frac{\Gamma(m+k) \Gamma(m-k-l)}{\Gamma(m-k-l-j) \Gamma(m+k-j)}-\frac{\Gamma(m+k+1) \Gamma(m-1-k-l)}{\Gamma(m-1-k-l-j) \Gamma(m+k+1-j)} \\
= & \frac{\Gamma(m+k) \Gamma(m-k-l)}{\Gamma(m-k-l-j) \Gamma(m+k-j)}\left[1-\frac{(m+k)(m-1-k-l-j)}{(m+k-j)(m-1-k-l)}\right] \\
= & \frac{\Gamma(m+k) \Gamma(m-k-l)}{\Gamma(m-k-l-j) \Gamma(m+k-j)} \cdot \frac{(2 k+1+l) j}{(m+k-j)(m-1-k-l)} \\
= & (2 k+1+l) \frac{j \Gamma(m+k) \Gamma(m-1-k-l)}{\Gamma(m-k-l-j) \Gamma(m+k+1-j)} .
\end{aligned}
$$


Therefore, combining (6.5) and (6.6) yields

$$
\begin{aligned}
& {\left[\prod_{j=0}^{l}\left((k+j+1 / 2)^{2}-(n-1)^{2} / 4-\Delta_{\mathbb{H}}\right)\right]^{-1} } \\
= & \frac{\pi^{-m}(\sinh \rho)^{2-2 m}}{(2 k+1+l) l 4 \Gamma(l)}\left[\frac{\Gamma(m+k) \Gamma(k+1)}{\Gamma(2 k+l+1)}\left(\sinh \frac{\rho}{2}\right)^{2 m-2 k-4}+\right. \\
& \left.(2 k+1+l) \sum_{j=1}^{m-2-k-l} \frac{j \Gamma(m+k) \Gamma(m-1-k-l) \Gamma(m-j-l)}{\Gamma(j+1) \Gamma(m-k-l-j) \Gamma(m+k+1-j)}\left(\sinh \frac{\rho}{2}\right)^{2 j+2 l-2}\right] . \\
= & \frac{\pi^{-m}(\sinh \rho)^{2-2 m}}{4 \Gamma(l+1)} \sum_{j=0}^{m-2-k-l} \frac{\Gamma(m+k) \Gamma(m-1-k-l) \Gamma(m-1-j-l)}{\Gamma(j+1) \Gamma(m-1-k-l-j) \Gamma(m+k-j)}\left(\sinh \frac{\rho}{2}\right)^{2 j+2 l}
\end{aligned}
$$

The desired result follows.

Lemma 6.3. Let $n=2 m$ and $1<k<n / 2$. The Green's function of $P_{k}$ satisfies

$$
\begin{aligned}
P_{k}^{-1}(\rho) & =\frac{4^{k-1}}{\gamma_{2 m}(2 k)(\sinh \rho)^{2 m-2}} \sum_{j=0}^{m-1-k} \frac{\Gamma(m)}{\Gamma(j+1) \Gamma(m-j)}\left(\sinh \frac{\rho}{2}\right)^{2 j+2 k-2} \\
& \leq \frac{1}{\gamma_{n}(2 k)}\left[\left(\frac{1}{2 \sinh \frac{\rho}{2}}\right)^{n-2 k}-\left(\frac{1}{2 \cosh \frac{\rho}{2}}\right)^{n-2 k}\right] .
\end{aligned}
$$

Proof. Choosing $k=0$ and $l=k$, we have

$$
\begin{aligned}
P_{k}^{-1} & =\left[\prod_{j=0}^{k-1}\left((j+1 / 2)^{2}-(n-1)^{2} / 4-\Delta_{\mathbb{H}}\right)\right]^{-1} \\
& =\frac{1}{4 \Gamma(k) \pi^{m}(\sinh \rho)^{2 m-2}} \sum_{j=0}^{m-1-k} \frac{\Gamma(m) \Gamma(m-k)}{\Gamma(j+1) \Gamma(m-j)}\left(\sinh \frac{\rho}{2}\right)^{2 j+2 k-2} \\
& =\frac{4^{k-1}}{\gamma_{2 m}(2 k)(\sinh \rho)^{2 m-2}} \sum_{j=0}^{m-1-k} \frac{\Gamma(m)}{\Gamma(j+1) \Gamma(m-j)}\left(\sinh \frac{\rho}{2}\right)^{2 j+2 k-2} .
\end{aligned}
$$


On the other hand,

$$
\begin{aligned}
& \frac{1}{\gamma_{n}(2 k)}\left[\left(\frac{1}{2 \sinh \frac{\rho}{2}}\right)^{n-2 k}-\left(\frac{1}{2 \cosh \frac{\rho}{2}}\right)^{n-2 k}\right] \\
= & \frac{4^{k-1}}{\gamma_{2 m}(2 k)(\sinh \rho)^{2 m-2}}\left[\left(\cosh \frac{\rho}{2}\right)^{2 m-2}\left(\sinh \frac{\rho}{2}\right)^{2 k-2}-\left(\cosh \frac{\rho}{2}\right)^{2 k-2}\left(\sinh \frac{\rho}{2}\right)^{2 m-2}\right] \\
= & \frac{4^{k-1}}{\gamma_{2 m}(2 k)(\sinh \rho)^{2 m-2}}\left[\left(1+\sinh ^{2} \frac{\rho}{2}\right)^{m-1}\left(\sinh \frac{\rho}{2}\right)^{2 k-2}-\right. \\
& \left.\left(1+\sinh ^{2} \frac{\rho}{2}\right)^{k-1}\left(\sinh \frac{\rho}{2}\right)^{2 m-2}\right] \\
= & \frac{4^{k-1}}{\gamma_{2 m}(2 k)(\sinh \rho)^{2 m-2}}\left[\sum_{j=0}^{m-1} \frac{\Gamma(m)}{\Gamma(j+1) \Gamma(m-j)}\left(\sinh \frac{\rho}{2}\right)^{2 j+2 k-2}-\right. \\
& \left.\sum_{j=0}^{k-1} \frac{\Gamma(k)}{\Gamma(j+1) \Gamma(k-j)}\left(\sinh \frac{\rho}{2}\right)^{2 j+2 m-2}\right] .
\end{aligned}
$$

Therefore,

$$
\begin{aligned}
& \frac{1}{\gamma_{n}(2 k)}\left[\left(\frac{1}{2 \sinh \frac{\rho}{2}}\right)^{n-2 k}-\left(\frac{1}{2 \cosh \frac{\rho}{2}}\right)^{n-2 k}\right]-P_{k}^{-1} \\
= & \frac{4^{k-1}}{\gamma_{2 m}(2 k)(\sinh \rho)^{2 m-2}}\left[\sum_{j=m-k}^{m-1} \frac{\Gamma(m)}{\Gamma(j+1) \Gamma(m-j)}\left(\sinh \frac{\rho}{2}\right)^{2 j+2 k-2}-\right. \\
& \left.\sum_{j=0}^{k-1} \frac{\Gamma(k)}{\Gamma(j+1) \Gamma(k-j)}\left(\sinh \frac{\rho}{2}\right)^{2 j+2 m-2}\right] . \\
= & \frac{4^{k-1}}{\gamma_{2 m}(2 k)(\sinh \rho)^{2 m-2}}\left[\sum_{j=0}^{k-1} \frac{\Gamma(m)}{\Gamma(m-k+j+1) \Gamma(k-j)}\left(\sinh \frac{\rho}{2}\right)^{2 j+2 m-2}-\right. \\
& \left.\sum_{j=0}^{k-1} \frac{\Gamma(k)}{\Gamma(j+1) \Gamma(k-j)}\left(\sinh \frac{\rho}{2}\right)^{2 j+2 m-2}\right] \\
= & \frac{4^{k-1}}{\gamma_{2 m}(2 k)(\sinh \rho)^{2 m-2}} \sum_{j=0}^{k-1} \frac{1}{\Gamma(k-j)}\left(\frac{\Gamma(m)}{\Gamma(m-k+j+1)}-\frac{\Gamma(k)}{\Gamma(j+1)}\right)\left(\sinh \frac{\rho}{2}\right)^{2 j+2 m-2} \\
\geq & 0 .
\end{aligned}
$$

This completes the proof of Lemma 6.3. 
6.2. Case 2: $n$ is odd. The proof depends on the following facts: if we denote by $h_{n}(t, \rho)$ the heat kernel of $n$-dimension hyperbolic space, then (see [17], page 178)

$$
h_{n}(t, \rho)=\sqrt{2} e^{\frac{2 n-1}{4} t} \int_{\rho}^{\infty} \frac{\sinh r}{\sqrt{\cosh r-\cosh \rho}} h_{n+1}(t, r) d r .
$$

Therefore, if we denote by $G_{n}(\rho, \lambda)\left(\lambda \geq-\frac{(n-1)^{2}}{4}\right)$ be the Green's function of $\lambda-\Delta_{\mathbb{H}}$ of dimension $n$, then by (6.11),

$$
\begin{aligned}
G_{n}(\rho, \lambda) & =\int_{0}^{\infty} e^{-\lambda t} h_{n}(t, \rho) d t \\
& =\sqrt{2} \int_{\rho}^{\infty} \frac{\sinh r}{\sqrt{\cosh r-\cosh \rho}}\left(\int_{0}^{\infty} e^{-\lambda t+\frac{2 n-1}{4} t} h_{n+1}(t, r) d t\right) d r \\
& =\sqrt{2} \int_{\rho}^{\infty} \frac{\sinh r}{\sqrt{\cosh r-\cosh \rho}} G_{n+1}(r, \lambda-(2 n-1) / 4) d r
\end{aligned}
$$

and thus

$$
\begin{aligned}
& G_{n}\left(\rho,(k+i+1 / 2)^{2}-(n-1)^{2} / 4\right) \\
= & \sqrt{2} \int_{\rho}^{\infty} \frac{\sinh r}{\sqrt{\cosh r-\cosh \rho}} G_{n+1}\left(r,(k+i+1 / 2)^{2}-n^{2} / 4\right) d r .
\end{aligned}
$$

By (6.13) and Lemma 6.2, we have that if $n=2 m-1$, then

$$
\begin{aligned}
& {\left[\prod_{j=0}^{l-1}\left((k+j+1 / 2)^{2}-(n-1)^{2} / 4-\Delta_{\mathbb{H}}\right)\right]^{-1} } \\
= & \sqrt{2} \int_{\rho}^{\infty}\left[\frac{1}{4 \Gamma(l) \pi^{m}(\sinh r)^{2 m-2}} \sum_{j=0}^{m-1-k-l} \frac{\Gamma(m+k) \Gamma(m-k-l) \Gamma(m-j-l)}{\Gamma(j+1) \Gamma(m-k-l-j) \Gamma(m+k-j)} .\right. \\
& \left.\left(\sinh \frac{r}{2}\right)^{2 j+2 l-2}\right] \frac{\sinh r}{\sqrt{\cosh r-\cosh \rho}} d r
\end{aligned}
$$

where $l \in\{1,2, \cdots, m-1\}$ and $k \in\{0,1, \cdots, m-1-l\}$.

Lemma 6.4. Let $n=2 m-1 \geq 3$ and $1<k<n / 2$. The Green's function of $P_{k}$ satisfies

$$
\begin{aligned}
P_{k}^{-1}(\rho) & =\frac{4^{k-1} \sqrt{2}}{\gamma_{2 m}(2 k)} \sum_{j=0}^{m-1-k} \frac{\Gamma(m)}{\Gamma(j+1) \Gamma(m-j)} \int_{\rho}^{\infty}\left(\sinh \frac{r}{2}\right)^{2 j+2 k-2} \frac{(\sinh r)^{3-2 m}}{\sqrt{\cosh r-\cosh \rho}} d r \\
& \leq \frac{1}{\gamma_{n}(2 k)}\left[\left(\frac{1}{2 \sinh \frac{\rho}{2}}\right)^{n-2 k}-\left(\frac{1}{2 \cosh \frac{\rho}{2}}\right)^{n-2 k}\right] .
\end{aligned}
$$


Proof. With the same argument as in the proof of Lemma 6.3, we have, by (6.14),

$$
\begin{aligned}
P_{k}^{-1}(\rho) & =\frac{4^{k-1} \sqrt{2}}{\gamma_{2 m}(2 k)} \sum_{j=0}^{m-1-k} \frac{\Gamma(m)}{\Gamma(j+1) \Gamma(m-j)} \int_{\rho}^{\infty}\left(\sinh \frac{r}{2}\right)^{2 j+2 k-2} \frac{(\sinh r)^{3-2 m}}{\sqrt{\cosh r-\cosh \rho}} d r \\
& \leq \frac{\sqrt{2}}{\gamma_{2 m}(2 k)} \int_{\rho}^{\infty}\left[\left(\frac{1}{2 \sinh \frac{\rho}{2}}\right)^{2 m-2 k}-\left(\frac{1}{2 \cosh \frac{\rho}{2}}\right)^{2 m-2 k}\right] \frac{\sinh r}{\sqrt{\cosh r-\cosh \rho}} d r
\end{aligned}
$$

Substituting $t=\sqrt{(\cosh r-\cosh \rho) / 2}$ yields

$$
\begin{aligned}
P_{k}^{-1}(\rho) & \leq \frac{1}{\gamma_{2 m}(2 k) 2^{2 m-2 k}} \int_{0}^{\infty}\left[\left(\frac{1}{t^{2}+\sinh ^{2} \frac{\rho}{2}}\right)^{m-k}-\left(\frac{1}{t^{2}+\cosh ^{2} \frac{\rho}{2}}\right)^{m-k}\right] 4 d t \\
& =\frac{4}{\gamma_{2 m}(2 k) 2^{2 m-2 k}} \cdot \frac{(2 m-2 k-3) ! ! \pi}{2 \cdot(2 m-2 k-2) ! !}\left[\left(\frac{1}{\sinh \frac{\rho}{2}}\right)^{2 m-2 k-1}-\left(\frac{1}{\cosh \frac{\rho}{2}}\right)^{2 m-2 k-1}\right] \\
& =\frac{1}{\gamma_{2 m-1}(2 k)}\left[\left(\frac{1}{2 \sinh \frac{\rho}{2}}\right)^{n-2 k}-\left(\frac{1}{2 \cosh \frac{\rho}{2}}\right)^{n-2 k}\right] .
\end{aligned}
$$

To get the first equality, we use the identity (see e.g. [22], page 324)

$$
\int_{0}^{\infty} \frac{d t}{\left(t^{2}+a^{2}\right)^{n}}=\frac{(2 n-3) ! !}{2 \cdot(2 n-2) ! !} \frac{\pi}{a^{2 n-1}}, \quad a>0 .
$$

The proof of Lemma 6.4 is thereby completed.

Proof of Theorem 1.10 Combining Lemma 6.3 and 6.4, Theorem 1.10 follows.

\section{REFERENCES}

[1] L.V. Ahlfors, Möbius Transformations in Several Dimensions, Ordway Professorship Lectures in Mathematics, University of Minnesota, School of Mathematics, Minneapolis, MN, 1981.

[2] J.-P. Anker, $L_{p}$ Fourier multipliers on Riemannian symmetric spaces of the noncompact type. Ann. of Math., 132(3) (1990)597-628.

[3] J.-P. Anker, L. Ji, Heat kernel and Green function estimates on noncompact symmetric spaces, Geom. Funct. Anal., 9 (1999) 1035-1091.

[4] T. Aubin, Espaces de Sobolev sur les variétés Riemanniennes. Bull. Sci. Math., 100(1976) 149-173.

[5] W. Beckner, Sharp Sobolev inequalities on the sphere and the Moser-Trudinger inequality. Ann. of Math. (2) 138 (1993), no. 1, 213-242.

[6] W. Beckner, On the Grushin operator and hyperbolic symmetry, Proc. Amer. Math. Soc., 129(2001), 1233-1246.

[7] W. Beckner, On Lie groups and hyperbolic symmetry-from Kunze-Stein phenomena to Riesz potentials. Nonlinear Anal. 126 (2015), 394-414.

[8] W. Beckner, Pitt's inequality with sharp convolution estimates, Proc. Amer. Math. Soc. 136 (2008), no. 5, 1871-1885.

[9] W. Beckner, Pitt's inequality and the fractional Laplacian: sharp error estimates. Forum Math. 24 (2012), no. 1, 177-209. 
[10] R.D. Benguria, R.L. Frank, M. Loss, The sharp constant in the Hardy-Sobolev-Maz'ya inequality in the three dimensional upper half space, Math. Res. Lett. 15 (2008) 613-622.

[11] H. Brezis, M. Marcus, Hardy's inequalities revisited, Dedicated to Ennio De Giorgi. Ann. Scuola Norm. Sup. Pisa Cl. Sci. (4) 25 (1997), no. 1-2, 217-237 (1998).

[12] H. Brezis, J. L. Vázquez, Blow-up solutions of some nonlinear elliptic problems, Rev. Mat. Univ. Complut. Madrid, 10, 1997, 443-469.

[13] J. Clerc and E. Stein, $L^{p}$-multipliers for noncompact symmetric spaces. Proc. Nat. Acad. Sci. U.S.A. 71 (1974), 3911-3912.

[14] A. Cotsiolis, N. K. Tavoularis, Best constants for Sobolev inequalities for higher order fractional derivatives, J. Math. Anal. Appl. 295 (2004), 225-236.

[15] E. Damek and F. Ricci, A class of nonsymmetric harmonic Riemannian spaces. Bull. Amer. Math. Soc. (N.S.) 27 (1992), no. 1, 139-142.

[16] E. B. Davies, N. Mandouvalos, Heat kernel bounds on hyperbolic space and Kleinian groups, Proc. London Math. Soc. (3) 52 (1988), 182-208.

[17] E. B. Davies, Heat kernels and spectral theory. Cambridge Tracts in Mathematics 92. Cambridge University Press, Cambridge 1989.

[18] A. Erdélyi, W. Magnus, F. Oberhettinger, F. G. Tricomi, Higher transcendental functions. Vols. I Based, in part, on notes left by Harry Bateman. McGraw-Hill Book Company, Inc., New York-Toronto-London 1953.

[19] S. Filippas, V. G. Maz'ya, A. Tertikas, Critical Hardy-Sobolev inequalities. J. Math. Pures Appl. 87, no.1, (2007), 37-56.

[20] R.L. Frank, M. Loss, Hardy-Sobolev-Maz'ya inequalities for arbitrary domains, J. Math. Pures Appl. 97 (2011) 39-54.

[21] A.R. Gover. Laplacian operators and Q-curvature on conformally Einstein manifolds. Math. Ann. 336 (2006), 311-334.

[22] I. S. Gradshteyn, L. M. Ryzhik, Table of Integrals, Series, and Products. 7th edition. Translation edited and with a preface by Alan Jeffrey and Daniel Zwillinger. Academic Press, Inc., San Diego, CA, 2007. Reproduction in P.R.China authorized by Elsevier (Singapore) Pte Ltd.

[23] C.R. Graham, R. Jenne, L.J. Mason, and G.A.J. Sparling, Conformally invariant powers of the Laplacian. I. Existence. Journal of the London Mathematical Society, (2)46 (1992), 557-565.

[24] A. Grigoryan, M. Noguchi, The heat kernel on hyperbolic space, Bull. London Math. Soc. 30 (1998), 643-650.

[25] E. Hebey, Nonlinear Analysis on Manifolds: Sobolev Spaces and Inequalities, Courant Lecture Notes in Mathematics Vol. 5, AMS, Providence, RI, 1999.

[26] E. Hebey, Sobolev spaces on Riemannian manifolds, Lecture Notes in Mathematics, Vol. 1635, SpringerVerlag, Berlin, 1996.

[27] S. Helgason, Groups and geometric analysis. Integral geometry, invariant differential operators, and spherical functions. Pure and Applied Mathematics. 113 Academic Press, 1984.

[28] S. Helgason, Geometric analysis on symmetric spaces. Second edition. Mathematical Surveys and Monographs, 39. American Mathematical Society, Providence, RI, 2008.

[29] Q. Hong, Sharp constant in third-order Hardy-Sobolev-Maz'ya inequality in the half space of dimension seven, to appear in International Math. Res. Notes.

[30] L. K. Hua, Starting with the Unit Circle, Springer-Verlag, Heidelberg, 1981.

[31] A. Ionescu, Singular integrals on symmetric spaces of real rank one. Duke Math. J. 114 (2002), no. 1, 101-122.

[32] A. Ionescu, Singular integrals on symmetric spaces. II. Trans. Amer. Math. Soc. 355 (2003), no. 8, 3359-3378.

[33] A. Juhl, Explicit formulas for GJMS-operators and Q-curvatures, Geom. Funct. Anal. 23(2013), 12781370. 
[34] N. Lam, G. Lu and L. Zhang, Factorizations and Hardy's type identities and inequalities on upper half spaces, to appear.

[35] J. Li, G. Lu, Q. Yang, Fourier analysis and optimal Hardy-Adams inequalities on hyperbolic spaces of any even dimension, Adv. Math., 333(2018), 350-385.

[36] J. Li, G. Lu and Q. Yang, Sharp Adams and Hardy-Adams inequalities of any fractional order on hyperbolic spaces of all dimensions, Preprint (2018).

[37] H.-Q. Li, Centered Hardy-Littlewood maximal function on hyperbolic spaces, $p>1$, arXiv:1304.3261v2 [math.CA].

[38] E. H. Lieb, Sharp constants in the Hardy-Littlewood-Sobolev and related inequalities. Ann. of Math. (2) 118 (1983), 349-374.

[39] C. Liu, L. Peng, Generalized Helgason-Fourier transforms associated to variants of the Laplace-Beltrami operators on the unit ball in $\mathbb{R}^{n}$, Indiana Univ. Math. J., 58, No. 3, (2009), 1457-1492.

[40] G. Liu, Sharp higher-order Sobolev inequalities in the hyperbolic space $\mathbb{H}^{n}$, Calc. Var. Partial Differential Equations 47, no. 3-4, (2013), 567-588

[41] G. Lu, Q. Yang, A sharp Trudinger-Moser inequality on any bounded and convex planar domain, Calc. Var. Partial Differ. Equ. 55: 153, 1-16(2016).

[42] G. Lu, Q. Yang, Sharp Hardy-Adams inequalities for bi-Laplacian on hyperbolic space of dimension four, Adv. Math. 319(2017) 567-598.

[43] G. Lu, Q. Yang, Paneitz operators on hyperbolic spaces and high order Hardy-Sobolev-Maz'ya inequalities on half spaces, arXiv:1703.08171, to appear in Amer. J. Math.

[44] G. Mancini, K. Sandeep, On a semilinear elliptic equation in $\mathbb{H}^{n}$, Ann. Scoula Norm. Sup. Pisa Cl. Sci. (5) Vol VII (2008), 635-671.

[45] H. Matsumoto, Closed form formulae for the heat kernels and the Green functions for the Laplacians on the symmetric spaces of rank one. Bull. Sci. Math. 125 (2001) 553-581.

[46] V.G. Maz'ya, Sobolev Spaces, Springer-Verlag, Berlin, 1985.

[47] V. G. Maz'ya, T. Shaposhnikova, A collection of sharp dilation invariant integral inequalities for differentiable functions, in: V. Maz'ya (Ed.), Sobolev Spaces in Mathematics I: Sobolev Type Inequalities, in: Int. Math. Ser., vol. 8, Springer, 2009, pp. 223-247.

[48] S. Paneitz, A quartic conformally covariant differential operator for arbitrary pseudo-Riemannian manifolds (summary). SIGMA Symmetry Integrability Geom. Methods Appl. 4 (2008), Paper 036, 3 pp.

[49] E. Stein, Some problems in harmonic analysis suggested by symmetric spaces and semi-simple groups. Actes du Congrs International des Mathmaticiens (Nice, 1970), Tome 1, pp. 173189. Gauthier-Villars, Paris, 1971.

[50] G. Talenti, Best constant in Sobolev inequality, Ann. Mat. Pura Appl., 110(1976), 353-372.

[51] A. Terras. Harmonic analysis on symmetric spaces and applications. I. Springer-Verlag, New York, 1985.

[52] A. Tertikas, K. Tintarev, On existence of minimizers for the Hardy-Sobolev-Maz'ya inequality, Ann. Mat. Pura Appl. (4) 186 (2007), 645-662.

[53] G. Wang, D. Ye, A Hardy-Moser-Trudinger inequality, Adv. Math. 230 (2012) 294-320.

[54] J. Wolf, Spaces of Constant Curvature, McGraw-Hill, New York (1967).

Department of Mathematics, University of Connecticut, Storrs, CT 06269, USA

E-mail address: guozhen.lu@uconn.edu

School of Mathematics and Statistics, Wuhan University, Wuhan, 430072, People's RePUBLIC OF CHINA

E-mail address: qhyang.math@whu.edu.cn; qhyang.math@gmail.com 